\title{
The Classical r-Matrix of AdS/CFT and its Lie Bialgebra Structure
}

\author{
Niklas Beisert ${ }^{1}$, Fabian Spill $^{2}$ \\ 1 Max-Planck-Institut für Gravitationsphysik, Albert-Einstein-Institut, Am Mühlenberg 1, \\ 14476, Potsdam, Germany. E-mail: nbeisert@aei.mpg.de \\ 2 Institut für Physik, Humboldt-Universität zu Berlin, Newtonstraße 15, D-12489 Berlin, Germany. \\ E-mail: spill@physik.hu-berlin.de
}

Received: 19 November 2007 / Accepted: 8 March 2008

Published online: 23 July 2008 - (C) The Author(s) 2008

\begin{abstract}
In this paper we investigate the algebraic structure of AdS/CFT in the strong-coupling limit. We propose an expression for the classical r-matrix with (deformed) $\mathfrak{u}(2 \mid 2)$ symmetry, which leads to a quasi-triangular Lie bialgebra as the underlying symmetry algebra. On the fundamental representation our r-matrix coincides with the classical limit of the quantum R-matrix.
\end{abstract}

\section{Introduction and Overview}

In the last five years since the discovery of integrable structures in the AdS/CFT correspondence [1-4] much progress has been made towards a complete solution of the two sides of the correspondence, superstring theory on $A d S_{5} \times S^{5}$ and $\mathcal{N}=4 \mathrm{SYM}$ theory, in the large- $N_{\mathrm{c}}$ limit. Indeed, assuming integrability, long-range Bethe Ansätze which fully describe the asymptotic spectrum of long operators or string states with large spins have been proposed [5]. These Bethe Ansätze use the factorised S-matrix [6] which describes the scattering of elementary excitations. For a generic integrable model, where our excitations, or magnons in the spin chain picture, carry momentum or rapidity $u$ it is not sufficient to work only with an ordinary semi-simple Lie algebra $\mathfrak{g}$, instead we usually work with the loop algebra $\mathfrak{g}\left[u, u^{-1}\right]$, its affinisation $\hat{g}$ or with related deformations of these structures to account for the spectral parameter. These deformations include (double) Yangians DY $(\mathfrak{g})$ and quantum affine algebras $\mathrm{U}_{q}(\hat{\mathfrak{g}})$, which lead to rational and trigonometric $\mathrm{S}$-matrices on evaluation representations, where the loop variable $u$ simply takes the value of some complex number and has the physical interpretation as the magnon rapidity. We can get these $S$-matrices on evaluation representations by solving the invariance equation $\left[\Delta\left(\mathfrak{J}^{A}\right), \mathcal{S}\right]=0$ for a minimal set of generators $\mathfrak{J}^{A}$ of the respective algebra. Alternatively they can be obtained from the representation of the universal R-matrix in case the underlying symmetry is a quasi-triangular Hopf algebra. This is the case for Yangians and quantum affine algebras. Note that in order to get a rapidity-dependent S-matrix it is usually not sufficient to demand invariance 
$\left[\Delta\left(\mathfrak{J}^{A}\right), \mathcal{S}\right]=0$ only for $\mathfrak{J}^{A} \in \mathfrak{g}$, but also for some $\mathfrak{J}^{A} \in \mathrm{DY}(\mathfrak{g})$, since the ordinary Lie generators usually do not depend on a spectral parameter.

For the AdS/CFT correspondence the symmetry algebra in question is $\mathfrak{p s u}(2,2 \mid 4)$, which is broken to $\mathfrak{u}(1) \ltimes \mathfrak{p s u}(2 \mid 2) \oplus \mathfrak{p s u}(2 \mid 2) \ltimes \mathfrak{u}(1)$ upon choosing a vacuum state in the Bethe ansatz. Due to the direct sum structure of this residual symmetry we can work with one copy of $\mathfrak{p s u}(2 \mid 2)$. Interestingly, $\mathfrak{p s u}(2 \mid 2)$ seems to be the only basic classical Lie superalgebra which allows for a non-trivial three-dimensional central extension $\mathfrak{p s u}(2 \mid 2) \ltimes \mathbb{R}^{3}$, and indeed this central extension seems necessary to derive the S-matrix for our model. This centrally extended algebra arises both on the gauge [7] and string theory [8] side of the correspondence. Interestingly, the S-matrix is already fully fixed [7] up to a factor by demanding only invariance under the Lie algebra generators of $\mathfrak{p s u}(2 \mid 2) \ltimes \mathbb{R}^{3}$, without referring to an additional loop algebra or something similar. One only needs to introduce an additional braiding element $[9,10]$ and identify the central charges and the braiding such that the central elements are all cocommutative. In that case, the S-matrix also depends on spectral parameters. The reason why it is fully fixed by the Lie algebra generators lies in the fact that the tensor product of two fundamental representations of $\mathfrak{p s u}(2 \mid 2) \ltimes \mathbb{R}^{3}$, in which the elementary magnons live, is generically irreducible [11]. Nevertheless, one might wonder if one can lift the $\mathfrak{p s u}(2 \mid 2) \ltimes$ $\mathbb{R}^{3}$ symmetry to some loop algebra or one of its deformations. It has been known for several years that there are some Yangian structures appearing on both sides of the correspondence [3,12-16]. Indeed, in [17] it has been shown that the S-matrix is invariant under the braided Yangian $Y\left(\mathfrak{p s u}(2 \mid 2) \ltimes \mathbb{R}^{3}\right)$. Recently there have been lots of other activities studying the encountered algebraic structures, see [18-21]. Since a Yangian usually has a universal R-matrix, it is natural to ask if the S-matrix on the fundamental evaluation representation arises as the representation of this R-matrix. In particular the overall phase of the S-matrix would directly follow. Due to quasi-triangularity, which is closely related to crossing symmetry, see [22], the dressing factor of the universal $\mathrm{R}$-matrix is constrained and perhaps fully fixed. This might lead to a derivation of the phase factor proposed in $[23,24]$ from first principles.

Even though there are standard methods how to construct the Yangian including its universal R-matrix for simple Lie algebras [25], there are several reasons why we cannot apply them in a simple fashion for our system. The main one seems to be the peculiar situation that our algebra $\mathfrak{p s u}(2 \mid 2) \ltimes \mathbb{R}^{3}$ has a non-trivial centre. This implies that the algebra is not simple and does not even admit a non-degenerate invariant supersymmetric bilinear form. ${ }^{1}$ Then there is the braiding element, which has to be related to the central charges in order to have cocommutativity on the centre. Furthermore, the ordinary Yangian spectral parameter also needs to be related to the central charges and the braiding. This makes the situation pretty complicated. Hence, investigating the classical bialgebra seems a promising way to study the underlying full quantum Hopf algebra and get an idea how to obtain its universal R-matrix. The crucial ingredient for the bialgebra is the classical r-matrix, which for our system was first investigated in [26]. In fact, a similar classical r-matrix, where the momentum scales differently with the coupling constant, was obtained directly from perturbation theory on the world sheet in [27], and in subsequent papers [28] the two-loop correction to the classical r-matrix have been computed. In [29], an algebraic expression for the classical r-matrix in the limit performed in [26] was written down which seems to indicate that the bialgebra is not the standard loop algebra of $\mathfrak{p s u}(2 \mid 2)$. This would probably imply that the universal R-matrix cannot be

\footnotetext{
1 One can obtain a non-degenerate form by adjoining outer automorphisms, which however do not live on the fundamental representation.
} 
obtained by the standard methods. However, in this paper we argue that the classical r-matrix of $\mathfrak{p s u}(2 \mid 2) \ltimes \mathbb{R}^{3}$ and the quasi-triangular Lie bialgebra which arises from this classical r-matrix are almost given by the standard formulae.

We begin by reviewing Lie bialgebras and their relation to Yangian doubles in Sect. 2. In Sect. 3 we apply these methods to $\mathfrak{p s u}(2 \mid 2) \ltimes \mathbb{R}^{3}$. We give explicit expressions for the corresponding Lie bialgebra and its classical r-matrix in Sect. 4 and relate it to standard algebras in Sect. 5. Different classical limits are investigated in Sect. 6. Finally, we provide first steps in lifting the classical bialgebra structure to the quantum case in Sect. 7.

\section{Classical r-Matrix and Lie Bialgebras}

In this section we will review the basic construction of bialgebras, classical r-matrices and how they arise as limiting cases of certain Hopf algebras. In particular, we are interested in the bialgebra structure of polynomials (or Laurent series) with values in a semi-simple Lie algebra, which lead upon quantisation to (double) Yangians, which in turn lead to rational solutions of the Yang-Baxter equation (YBE). We will deal only with the case of simple Lie algebras; the interested reader will find more details and proofs for this case in the textbook of Chari and Pressley [30] or in Drinfeld's original report [31]. For later purposes let us note that the generalisation to the case of Lie superalgebras as well as to non-simple Lie algebras; and superalgebras is straightforward provided they allow for a non-degenerate supersymmetric invariant bilinear form.

\subsection{R-matrix and double Yangian.}

Double Yangian. Consider a semi-simple Lie algebra $\mathfrak{g}$ spanned by the generators $\mathfrak{J}^{A}$ obeying the Lie bracket

$$
\left[\mathfrak{J}^{A}, \mathfrak{J}^{B}\right]=F_{C}^{A B} \mathfrak{J}^{C}
$$

with the structure constants $F_{C}^{A B}$. Define furthermore the Cartan-Killing matrix $C^{A B} \sim$ $F_{D}^{A C} F_{C}^{B D}$, its inverse $C_{A B}$ and the conjugated structure constants $F_{B C}^{A}=F_{B}^{A D} C_{D C}$.

Then the double Yangian DY $(\mathfrak{g})$ is a deformation of the universal enveloping algebra $\mathrm{U}\left(\mathfrak{g}\left[u, u^{-1}\right]\right)$ of the loop algebra $\mathfrak{g}\left[u, u^{-1}\right]$. It is generated by the level- $n$ generators $\mathfrak{J}_{n}^{A}$, $n \in \mathbb{Z}$, with level-zero defined to span the Lie algebra, $\mathfrak{J}_{0}^{A}=\mathfrak{J}^{A}$. The commutation relations of these generators read

$$
\left[\mathfrak{J}_{m}^{A}, \mathfrak{J}_{n}^{B}\right]=F_{C}^{A B} \mathfrak{J}_{n+m}^{C}+\mathcal{O}(\hbar)
$$

where $\hbar$ is the deformation parameter. The precise form of the deformations for the algebra does not appear very enlightening, and we have not made it explicit here. Their coproduct takes the standard form

$$
\Delta\left(\mathfrak{J}_{n}^{A}\right)=\mathfrak{J}_{n}^{A} \otimes 1+1 \otimes \mathfrak{J}_{n}^{A}+\hbar \sum_{m=0}^{n-1} \frac{1}{2} F_{B C}^{A} \mathfrak{J}_{n-1-m}^{B} \otimes \mathfrak{J}_{m}^{C}+\mathcal{O}\left(\hbar^{2}\right)
$$


Universal R-matrix. The double Yangian is quasi-triangular, it has been constructed as a quantum double of the original Yangian in [25]. This means it has an R-matrix $\mathcal{R} \in \mathrm{DY}(\mathfrak{g}) \otimes \mathrm{DY}(\mathfrak{g})$ obeying the cocommutativity relation

$$
\widetilde{\Delta}\left(\mathfrak{J}_{n}^{A}\right) \mathcal{R}=\mathcal{R} \Delta\left(\mathfrak{J}_{n}^{A}\right)
$$

with $\widetilde{\Delta}:=\mathcal{P} \circ \Delta$ being the opposite coproduct and $\mathcal{P}$ the permutation operator. Additional relations ensure that the YBE holds. We neither spell out these relations nor the explicit form of the universal R-matrix as we do not need them in what follows.

Evaluation representations. Often one considers evaluation representations of the Yangian. These representations are most relevant for integrable spin chains and most transparent. On a state $|u\rangle$ an evaluation representation of the double Yangian is defined by the action

$$
\mathfrak{J}_{n}^{A}|u\rangle=u^{n} \mathfrak{J}_{0}^{A}|u\rangle+\mathcal{O}(\hbar) \text { i.e. } \mathfrak{J}_{n}^{A} \simeq u^{n} \mathfrak{J}_{0}^{A}+\mathcal{O}(\hbar) .
$$

The representation of $\mathcal{R}$ on a state $\left|u_{1}\right\rangle \otimes\left|u_{2}\right\rangle$ then becomes the matrix-valued function $R\left(u_{1}, u_{2}\right)$ which is typically of a difference form $R\left(u_{1}-u_{2}\right)$, and leads to rational solutions of YBE. For invariance of $R\left(u_{1}, u_{2}\right)$ one merely needs to check invariance under $\mathfrak{J}_{0}^{A}$ and $\mathfrak{J}_{1}^{A}$ for invariance under $\mathfrak{J}_{n}^{A}$ follows from an identity

$$
\Delta\left(\mathfrak{J}_{n}^{A}\right) \simeq \frac{u_{1}^{n-1}-u_{2}^{n-1}}{u_{1}^{-1}-u_{2}^{-1}} \Delta\left(\mathfrak{J}_{0}^{A}\right)+\frac{u_{1}^{n}-u_{2}^{n}}{u_{1}-u_{2}} \Delta\left(\mathfrak{J}_{1}^{A}\right)+\mathcal{O}(\hbar)
$$

which holds for evaluation representations.

\subsection{Classical limit and Lie bialgebra.}

Classical Limit. Now let us consider the classical limit of the above algebra where we restrict to the first order in $\hbar$ everywhere. We first expand the coproduct and opposite coproduct

$$
\Delta=\Delta_{0}+\hbar \Delta_{1}+\mathcal{O}\left(\hbar^{2}\right), \quad \widetilde{\Delta}=\Delta_{0}+\hbar \widetilde{\Delta}_{1}+\mathcal{O}\left(\hbar^{2}\right) .
$$

The classical r-matrix is obtained from the quantum R-matrix by expansion in the deformation parameter $\hbar$,

$$
\mathcal{R}=1 \otimes 1+\hbar r+\mathcal{O}(\hbar)^{2}
$$

By substituting these two expressions into the quasi-cocommutativity (2.4) relation we obtain

$$
\left[\Delta_{0}\left(\mathfrak{J}_{n}^{A}\right), r\right]=\Delta_{1}\left(\mathfrak{J}_{n}^{A}\right)-\widetilde{\Delta}_{1}\left(\mathfrak{J}_{n}^{A}\right) .
$$

Similarly, if $\mathcal{R}$ satisfies the quantum YBE $\mathcal{R}_{12} \mathcal{R}_{13} \mathcal{R}_{23}=\mathcal{R}_{23} \mathcal{R}_{13} \mathcal{R}_{12}$, it is straightforward to check that the classical r-matrix will satisfy

$$
[[r, r]]:=\left[r_{12}, r_{13}\right]+\left[r_{12}, r_{23}\right]+\left[r_{13}, r_{23}\right]=0,
$$

which is called the classical Yang-Baxter equation (CYBE). 
Lie bialgebras. The above expansion can be cast into the framework of a Lie bialgebra. In general, a Lie bialgebra is a Lie algebra $\mathfrak{g}$ equipped with an antisymmetric linear map, called the cobracket,

$$
\delta: \mathfrak{g} \rightarrow \mathfrak{g} \otimes \mathfrak{g}
$$

such that the dual map $\delta^{*}: \mathfrak{g}^{*} \otimes \mathfrak{g}^{*} \rightarrow \mathfrak{g}^{*}$ is an ordinary Lie bracket. This means that if $\left(F^{*}\right)_{A B}{ }^{C}$ are structure constants of the cobracket, i.e. $\delta \mathfrak{J}^{A}=\left(F^{*}\right)_{B C}{ }_{B} \mathfrak{J}^{B} \otimes \mathfrak{J}^{C}$, then the same constants define the Lie bracket of $\mathfrak{g}^{*}$ for the corresponding dual basis, $\left[\mathfrak{J}_{A}, \mathfrak{J}_{B}\right]=\left(F^{*}\right)_{A B}{ }^{C} \mathfrak{J}_{C}$. Similarly, the structure constants of $\mathfrak{g}$ define a cobracket on $\mathfrak{g}^{*}$. Furthermore the cobracket $\delta$ is a cocycle, i.e.

$$
\delta\left(\left[\mathfrak{J}_{1}, \mathfrak{J}_{2}\right]\right)=\left[\mathfrak{J}_{1}, \delta\left(\mathfrak{J}_{2}\right)\right]-\left[\mathfrak{J}_{2}, \delta\left(\mathfrak{J}_{1}\right)\right],
$$

where one extends the Lie bracket canonically to the space $\mathfrak{g} \otimes \mathfrak{g}$ by defining (with proper signs due to fermi statistics implicit)

$$
\left[\mathfrak{J}_{1}, \mathfrak{J}_{2} \otimes \mathfrak{J}_{3}\right]=-\left[\mathfrak{J}_{2} \otimes \mathfrak{J}_{3}, \mathfrak{J}_{1}\right]:=\left[\mathfrak{J}_{1}, \mathfrak{J}_{2}\right] \otimes \mathfrak{J}_{3}+\mathfrak{J}_{2} \otimes\left[\mathfrak{J}_{1}, \mathfrak{J}_{3}\right] .
$$

We will be especially interested in coboundary bialgebras where the cobracket is obtained by commuting with a classical r-matrix $r$,

$$
\delta(\mathfrak{J})=[\mathfrak{J}, r] .
$$

The properties of the Lie bialgebra are satisfied if $[[r, r]]$, cf. (2.10), is invariant under the Lie algebra. In particular, if the r-matrix satisfies the classical Yang-Baxter equation $[[r, r]]=0$ the Lie bialgebra is called quasi-triangular.

The relation (2.14) matches Eq. (2.9) if we relate the cobracket as

$$
\delta\left(\mathfrak{J}_{n}^{A}\right)=\Delta_{1}\left(\mathfrak{J}_{n}^{A}\right)-\widetilde{\Delta}_{1}\left(\mathfrak{J}_{n}^{A}\right)
$$

for according to (2.13) we have $\left[\Delta_{0}\left(\mathfrak{J}_{n}^{A}\right), r\right]=\left[\mathfrak{J}_{n}^{A}, r\right]$. Note that our cobracket $(2.15)$ is obviously antisymmetric. Thus we have now formulated the relations in the classical limit purely in terms of a quasi-triangular Lie bialgebra.

In fact, what we presented in this paragraph is quite generic: whenever we have a quasi-triangular Hopf algebra which is a deformation of a universal enveloping algebra, we obtain a corresponding quasi-triangular Lie bialgebra by considering the properties of the Hopf algebra at the lowest orders in the deformation parameter $\hbar$, see [30].

Loop Algebra. The classical limit of a double Yangian leads to a Lie bialgebra based on the loop algebra $\mathfrak{g}\left[u, u^{-1}\right]$ of $\mathfrak{g}$. It has the standard bracket

$$
\left[\mathfrak{J}_{m}^{A}, \mathfrak{J}_{n}^{B}\right]=F_{C}^{A B} \mathfrak{J}_{n+m}^{C} .
$$

The cobracket is defined as

$$
\delta\left(\mathfrak{J}_{n}^{A}\right)=\frac{1}{2} F_{B C}^{A} \sum_{m=0}^{n-1} \mathfrak{J}_{n-1-m}^{B} \wedge \mathfrak{J}_{m}^{C},
$$

with the antisymmetric tensor product

$$
\mathfrak{J}_{1} \wedge \mathfrak{J}_{2}:=\mathfrak{J}_{1} \otimes \mathfrak{J}_{2}-\mathfrak{J}_{2} \otimes \mathfrak{J}_{1}
$$


It is not hard to confirm that the antisymmetric classical r-matrix

$$
r=\sum_{n=0}^{\infty} \frac{1}{2} C_{C D} \mathfrak{J}_{-1-n}^{C} \wedge \mathfrak{J}_{n}^{D}
$$

obeys the relation (2.14). Furthermore, $[[r, r]]$ is algebra-invariant and therefore the bialgebra is coboundary. In order to satisfy the CYBE $[[r, r]]=0$ we have to choose an asymmetric form for the r-matrix

$$
r=\sum_{n=0}^{\infty} C_{C D} \mathfrak{J}_{-1-n}^{C} \otimes \mathfrak{J}_{n}^{D} \quad \text { or } \quad r=-\sum_{n=0}^{\infty} C_{C D} \mathfrak{J}_{n}^{C} \otimes \mathfrak{J}_{-1-n}^{D} .
$$

In both cases the Lie bialgebra is quasi-triangular.

Evaluation representations. Consider now evaluation representations as above in (2.5). The representation of the r-matrix on a state $\left|u_{1}\right\rangle \otimes\left|u_{2}\right\rangle$ becomes

$$
r \simeq r\left(u_{1}, u_{2}\right)=\frac{C_{C D} \mathfrak{J}_{0}^{C} \otimes \mathfrak{J}_{0}^{D}}{u_{1}-u_{2}} .
$$

In fact all three forms (2.19), (2.20) are equivalent up to contact terms at $u_{1}=u_{2}$. The above action is proportional to the quadratic Casimir operator of the Lie algebra at level-0 and therefore obviously

$$
\left[\mathfrak{J}_{0}^{A}, r\right] \simeq 0=\delta\left(\mathfrak{J}_{0}^{A}\right) .
$$

For the level-one generator $\mathfrak{J}_{1}^{A}$ one also finds that the coboundary relation holds:

$$
\left[\mathfrak{J}_{1}^{A}, r\right] \simeq F_{B C}^{A} \mathfrak{J}_{0}^{B} \otimes \mathfrak{J}_{0}^{C}=\delta\left(\mathfrak{J}_{1}^{A}\right)
$$

For evaluation representations, the coboundary property for the remaining generator $\mathfrak{J}_{n}^{A}$ follows from the level-zero and level-one relations by means of the identity

$$
\delta\left(\mathfrak{J}_{n}^{A}\right) \simeq \frac{u_{1}^{n-1}-u_{2}^{n-1}}{u_{1}^{-1}-u_{2}^{-1}} \delta\left(\mathfrak{J}_{0}^{A}\right)+\frac{u_{1}^{n}-u_{2}^{n}}{u_{1}-u_{2}} \delta\left(\mathfrak{J}_{1}^{A}\right) .
$$

\section{Centrally Extended $\mathfrak{s u}(2 \mid 2)$}

3.1. Yangian double. We would like to understand the R-matrix that appears in the context of AdS/CFT on an algebraic level. Its symmetry is based on centrally extended $\mathfrak{s u}(2 \mid 2)$ symmetry $[32,7]$

$$
\mathfrak{h}:=\mathfrak{s u}(2 \mid 2) \ltimes \mathbb{R}^{2}=\mathfrak{p s u}(2 \mid 2) \ltimes \mathbb{R}^{3},
$$

and it acts on two four-dimensional fundamental representations of $\mathfrak{h}$. It is generated by the $\mathfrak{s u}(2) \times \mathfrak{s u}(2)$ generators $\mathfrak{R}_{b}^{a}, \mathfrak{L}^{\alpha}{ }_{\beta}$, the supercharges $\mathfrak{Q}^{\alpha}{ }_{b}, \mathfrak{S}^{a}{ }_{\beta}$ and the central charges $\mathfrak{C}, \mathfrak{P}, \mathfrak{K}$. Where appropriate, we shall use the collective symbol $\mathfrak{J}^{A}$ for these generators. The R-matrix also displays Yangian symmetry $Y(\mathfrak{h})$ [17] and by means of (2.6) double Yangian symmetry DY(h). The level- $n$ generators corresponding to $\mathfrak{J}^{A}$ shall be denoted by $\mathfrak{J}_{n}^{A}$. 
Commutators. The Lie brackets of the $\mathfrak{s u}(2) \times \mathfrak{s u}(2)$ generators take the standard form

$$
\begin{aligned}
{\left[\mathfrak{R}^{a}{ }_{b}, \mathfrak{R}_{d}^{c}{ }_{d}\right] } & =\delta_{b}^{c} \mathfrak{R}^{a}{ }_{d}-\delta_{d}^{a} \mathfrak{R}^{c}{ }_{b}, & {\left[\mathfrak{L}^{\alpha}{ }_{\beta}, \mathfrak{L}^{\gamma}{ }_{\delta}\right] } & =\delta_{\beta}^{\gamma} \mathfrak{L}^{\alpha}{ }_{\delta}-\delta_{\delta}^{\alpha} \mathfrak{L}_{\beta}^{\gamma}, \\
{\left[\mathfrak{R}_{b}{ }_{b}, \mathfrak{Q}^{\gamma}{ }_{d}\right] } & =-\delta_{d}^{a} \mathfrak{Q}^{\gamma}{ }_{b}+\frac{1}{2} \delta_{b}^{a} \mathfrak{Q}^{\gamma}{ }_{d}, & {\left[\mathfrak{L}^{\alpha}{ }_{\beta}, \mathfrak{Q}^{\gamma}{ }_{d}\right] } & =+\delta_{\beta}^{\gamma} \mathfrak{Q}^{\alpha}{ }_{d}-\frac{1}{2} \delta_{\beta}^{\alpha} \mathfrak{Q}^{\gamma}{ }_{d}, \\
{\left[\mathfrak{R}^{a}{ }_{b}, \mathfrak{S}^{c}{ }_{\delta}\right] } & =+\delta_{b}^{c} \mathfrak{S}^{a}{ }_{\delta}-\frac{1}{2} \delta_{b}^{a} \mathfrak{S}^{c}{ }_{\delta}, & {\left[\mathfrak{L}^{\alpha}{ }_{\beta}, \mathfrak{S}^{c}{ }_{\delta}\right] } & =-\delta_{\delta}^{\alpha} \mathfrak{S}^{c}{ }_{\beta}+\frac{1}{2} \delta_{\beta}^{\alpha} \mathfrak{S}^{c}{ }_{\delta} .
\end{aligned}
$$

The Lie brackets of two supercharges yield

$$
\begin{aligned}
\left\{\mathfrak{Q}^{\alpha}{ }_{b}, \mathfrak{S}^{c}{ }_{\delta}\right\} & =\delta_{b}^{c} \mathfrak{L}^{\alpha}{ }_{\delta}+\delta_{\delta}^{\alpha} \mathfrak{R}^{c}{ }_{b}+\delta_{b}^{c} \delta_{\delta}^{\alpha} \mathfrak{C}, \\
\left\{\mathfrak{Q}^{\alpha}{ }_{b}, \mathfrak{Q}^{\gamma}{ }_{d}\right\} & =\varepsilon^{\alpha \gamma} \varepsilon_{b d} \mathfrak{P}, \\
\left\{\mathfrak{S}^{a}{ }_{\beta}, \mathfrak{S}^{c}{ }_{\delta}\right\} & =\varepsilon^{a c} \varepsilon_{\beta \delta} \mathfrak{K} .
\end{aligned}
$$

The remaining Lie brackets vanish. Again, we do not write the commutators of the level-one generators explicitly.

Coproduct. For the coproduct one should introduce a non-trivial braiding $[9,10,17]$

$$
\begin{aligned}
\Delta\left(\mathfrak{J}_{n}^{A}\right) & =\mathfrak{J}_{n}^{A} \otimes 1+\mathcal{U}^{[A]} \otimes \mathfrak{J}_{n}^{A}+\frac{1}{2} g^{-1} F_{B C}^{A} \sum_{k=0}^{n-1} \mathfrak{J}_{k}^{B} \mathcal{U}^{[C]} \otimes \mathfrak{J}_{n-1-k}^{C}+\mathcal{O}\left(g^{-2}\right), \\
\Delta(\mathcal{U}) & =\mathcal{U} \otimes \mathcal{U},
\end{aligned}
$$

with some abelian generator $\mathcal{U}$ (a priori unrelated to the algebra) and the grading

$$
[\mathfrak{K}]=-2, \quad[\mathfrak{S}]=-1, \quad[\mathfrak{R}]=[\mathfrak{L}]=[\mathfrak{C}]=0, \quad[\mathfrak{Q}]=+1, \quad[\mathfrak{P}]=+2 .
$$

This "braid charge" is proportional to the charge under the external $\mathfrak{u}(1)$ automorphism $\overline{\mathfrak{B}}$ acting as $\left[\overline{\mathfrak{B}}, \mathfrak{J}^{A}\right]=[A] \mathfrak{J}^{A}$, and therefore the coproduct is compatible with the algebra relations.

To achieve a quasi-cocommutative algebra, the central charges $\mathfrak{P}_{0}, \mathfrak{K}_{0}, \mathfrak{P}_{1}, \mathfrak{K}_{1}$ must be identified with the braiding factor $\mathcal{U}$ and the central charge $\mathfrak{C}_{0}$ as follows $[10,17]^{2}$

$$
\begin{array}{ll}
\mathfrak{P}_{0}=g \alpha\left(1-\mathcal{U}^{+2}\right), & \mathfrak{K}_{0}=g \alpha^{-1}\left(1-\mathcal{U}^{-2}\right), \\
\mathfrak{P}_{1}=\alpha \mathfrak{C}_{0}\left(1+\mathcal{U}^{+2}\right), & \mathfrak{K}_{1}=-\alpha^{-1} \mathfrak{C}_{0}\left(1+\mathcal{U}^{-2}\right) .
\end{array}
$$

From these identifications it follows that the evaluation parameter $i u$ for any evaluation representation is fixed in terms of the eigenvalues of $\mathfrak{C}$ and $\mathcal{U}$,

$$
\mathfrak{J}_{n}^{A} \simeq(i u)^{n} \mathfrak{J}_{0}^{A}, \quad i u \simeq g^{-1} \mathfrak{C}_{0} \frac{1+\mathcal{U}^{+2}}{1-\mathcal{U}^{+2}}
$$

\footnotetext{
2 We set the inessential shift parameter $u_{0}$ in [17] to zero.
} 
Fundamental representation. The algebra $\mathfrak{h}$ has a four-dimensional representation [7] which we will call fundamental. The corresponding multiplet has two bosonic states $\left|\phi^{a}\right\rangle$ and two fermionic states $\left|\psi^{\alpha}\right\rangle$. The action of the two sets of $\mathfrak{s u}(2)$ generators has to be canonical

$$
\mathfrak{R}_{b}^{a}{ }_{b}\left|\phi^{c}\right\rangle=\delta_{b}^{c}\left|\phi^{a}\right\rangle-\frac{1}{2} \delta_{b}^{a}\left|\phi^{c}\right\rangle, \quad \mathfrak{L}^{\alpha}{ }_{\beta}\left|\psi^{\gamma}\right\rangle=\delta_{\beta}^{\gamma}\left|\psi^{\alpha}\right\rangle-\frac{1}{2} \delta_{\beta}^{\alpha}\left|\psi^{\gamma}\right\rangle
$$

The supersymmetry generators must also act in a manifestly $\mathfrak{s u}(2) \times \mathfrak{s u}(2)$ covariant way:

$$
\begin{array}{ll}
\mathfrak{Q}^{\alpha}{ }_{a}\left|\phi^{b}\right\rangle=a \delta_{a}^{b}\left|\psi^{\alpha}\right\rangle, & \mathfrak{Q}^{\alpha}{ }_{a}\left|\psi^{\beta}\right\rangle=b \varepsilon^{\alpha \beta} \varepsilon_{a b}\left|\phi^{b}\right\rangle, \\
\mathfrak{S}^{a}{ }_{\alpha}\left|\phi^{b}\right\rangle=c \varepsilon^{a b} \varepsilon_{\alpha \beta}\left|\psi^{\beta}\right\rangle, & \mathfrak{S}^{a}{ }_{\alpha}\left|\psi^{\beta}\right\rangle=d \delta_{\alpha}^{\beta}\left|\phi^{a}\right\rangle .
\end{array}
$$

We can write the four parameters $a, b, c, d$ using the parameters $x^{ \pm}, \gamma$ and the constants $g, \alpha$ as

$$
a=\sqrt{g} \gamma, \quad b=\sqrt{g} \frac{\alpha}{\gamma}\left(1-\frac{x^{+}}{x^{-}}\right), \quad c=\sqrt{g} \frac{i \gamma}{\alpha x^{+}}, \quad d=\sqrt{g} \frac{x^{+}}{i \gamma}\left(1-\frac{x^{-}}{x^{+}}\right) .
$$

The parameters $x^{ \pm}$(together with $\gamma$ ) label the representation ${ }^{3}$ and they must obey the constraint

$$
x^{+}+\frac{1}{x^{+}}-x^{-}-\frac{1}{x^{-}}=\frac{i}{g} .
$$

The three central charges $\mathfrak{C}, \mathfrak{P}, \mathfrak{K}$ and $\mathcal{U}$ are represented by the values $C, P, K$ and $U$ which read

$$
\begin{gathered}
C=\frac{1}{2} \frac{1+1 / x^{+} x^{-}}{1-1 / x^{+} x^{-}}, \quad P=g \alpha\left(1-\frac{x^{+}}{x^{-}}\right), \quad K=\frac{g}{\alpha}\left(1-\frac{x^{-}}{x^{+}}\right), \\
U=e^{i p / 2}=\sqrt{\frac{x^{+}}{x^{-}}} .
\end{gathered}
$$

The coefficient $U$ is most immediately related to the particle momentum $p$ used in the scattering matrix by $U=e^{i p / 2}$. These eigenvalues obey the quadratic relation $C^{2}-P K=\frac{1}{4}$ by virtue of (3.11). Note that the corresponding quadratic combination of central charges $\mathfrak{C}^{2}-\mathfrak{P} \mathfrak{K}$ is singled out by being invariant under the external $\mathfrak{s l}(2)$ automorphism of $\mathfrak{h}$, see Sect. 5.2.

The representation of the Yangian DY $(\mathfrak{h})$ is of evaluation type $\mathfrak{J}_{n}^{A} \simeq(i u)^{n} \mathfrak{J}_{0}^{A}$ [17]. The evaluation parameter $u$ is related to the $x^{ \pm}$parameters by

$$
u=x^{+}+\frac{1}{x^{+}}-\frac{i}{2 g}=x^{-}+\frac{1}{x^{-}}+\frac{i}{2 g}=\frac{1}{2}\left(x^{+}+x^{-}\right)\left(1+1 / x^{+} x^{-}\right) .
$$

\footnotetext{
${ }^{3}$ For a hermitian representation we should set $|\gamma|=\left|\sqrt{-i x^{+}+i x^{-}}\right|$and $|\alpha|=1$.
} 
Fundamental R-matrix. In $[7,11]$ an S-matrix acting on the tensor product of two fundamental representations was derived. It was constructed by imposing invariance under the algebra $\mathfrak{h}[7,11]$, and it was shown to be invariant under Yangian generators [17]

$$
\left[\Delta\left(\mathfrak{J}_{n}^{A}\right), \mathcal{S}\right]=0 .
$$

The S-matrix also satisfies the YBE [7,11]. We will not reproduce the result here, it is given in [11]. Note that we have to fix the parameters $\xi=U=\sqrt{x^{+} / x^{-}}$in order to make the action of the generators compatible with the coproduct (3.4). ${ }^{4}$

From the S-matrix we can read off a fundamental R-matrix

$$
\mathcal{S}=\mathcal{P} \mathcal{R},
$$

where $\mathcal{P}$ is a (graded) permutation operator. Upon this identification, invariance of the $\mathrm{S}$-matrix in (3.14) is equivalent to quasi-cocommutativity (2.4) of the R-matrix.

The next step would be to construct the universal R-matrix for the algebra $\mathfrak{h}$. Our centrally extended algebra $\mathfrak{h}$ is however not semi-simple and therefore the standard construction of the universal R-matrix cannot be applied. The main reason for the failure is that the Cartan-Killing matrix $C^{A B}$ is singular and its inverse $C_{A B}$, which plays an important role in the construction, does not exist. Similarly, for the standard construction of the classical r-matrix one would need the quadratic Casimir $\frac{1}{2} C_{A B} \mathfrak{J}^{A} \mathfrak{J}^{B}$ which does not exist for our algebra. 5

The R-matrix has one overall phase factor $S^{0}$,

$$
S_{12}^{0}=\exp \left(i \theta_{21}\right) \sqrt{\frac{1-1 / x_{2}^{+} x_{1}^{-}}{1-1 / x_{2}^{-} x_{1}^{+}} \frac{x_{2}^{-}-x_{1}^{+}}{x_{2}^{+}-x_{1}^{-}}},
$$

where $\theta$ is the so-called dressing phase. The phase cannot be determined from quasicocommutativity. Quasi-triangularity, however, imposes some constraint which is believed to give the crossing symmetry relation found in [22]. In [23,24] a proposal for a crossing-symmetric phase was made. The proposal is fully consistent with perturbative results from gauge theory [14] and from string theory [34-37].

For simplicity we shall choose a specific dressing factor which does not obey crossing. It turns out that the light cone string S-matrix [38] leads to convenient and symmetric expressions. The dressing factor for this case is

$$
S_{12}^{0}=\sqrt{\sqrt{\frac{x_{1}^{+} x_{2}^{-}}{x_{1}^{-} x_{2}^{+}}} \frac{x_{2}^{-}-x_{1}^{+}}{x_{2}^{+}-x_{1}^{-}}}, \quad\left(A_{12}\right)^{2}=\sqrt{\frac{x_{1}^{+} x_{2}^{-}}{x_{1}^{-} x_{2}^{+}}} \frac{x_{2}^{+}-x_{1}^{-}}{x_{2}^{-}-x_{1}^{+}} .
$$

At leading order at strong coupling (on which we will focus our attention in the remainder of the paper) this phase factor agrees with the correct physical result up to a term which can be absorbed into the definition of the length of the string. Another useful choice is

$$
S_{12}^{0}=\exp \left(i \theta_{21}\right) \sqrt{\frac{x_{1}^{+} x_{2}^{-}}{x_{1}^{-} x_{2}^{+}} \frac{1-1 / x_{2}^{+} x_{1}^{-}}{1-1 / x_{2}^{-} x_{1}^{+}} \frac{x_{2}^{-}-x_{1}^{+}}{x_{2}^{+}-x_{1}^{-}}},
$$

which differs from (3.16) by some factors of the particle momenta leading to a redefinition of the length of a state.

\footnotetext{
4 This identification removes all braiding factors from the S-matrix in [11] which will thus satisfy the standard Yang-Baxter (matrix) equation, see also [7, 18,33].

5 Due to the deformed coproduct for Lie generators, this is actually not what one really wants.
} 
3.2. Classical limit and Lie bialgebra. A suitable classical limit for the above S-matrix is the limit where the particle momenta $p$ scale like $\hbar$ while the coupling constant $g$ approaches infinity like $1 / \hbar$. This limit is well-known as the (near) plane wave limit [39-41,1] (for finitely many excitations above the vacuum) or as the classical limit for spinning strings [42-45] (for coherent states of infinitely many excitations). In this limit the evaluation parameter $u$ becomes large as $1 / \hbar$ as for typical classical limits.

One may also consider a different limit $u \sim 1 / \hbar$ but $g \sim 1 / \hbar^{\kappa}$ with adjustable $\kappa$. The standard classical limit corresponds to $\kappa=1$. For $\kappa>1$ it turns out that limit of the R-matrix is not of the form $\mathcal{R}=1 \otimes 1+\mathcal{O}(\hbar)$. Conversely, for $\kappa<1$ the R-matrix has a classical limit $\mathcal{R}=1 \otimes 1+\mathcal{O}(\hbar)$, but with an r-matrix which is a twisted version of the standard $\mathfrak{u}(2 \mid 2)$ r-matrix. We shall review this case in Sect. 6 .

Lie bialgebra. The classical limit described above is the limit $g \rightarrow \infty$, i.e. the quantum parameter is $\hbar=g^{-1}$, while assuming that

$$
\mathcal{U}=\exp \left(\frac{i}{2} g^{-1} \mathfrak{D}\right)
$$

with some finite abelian generator $\mathfrak{D}$. This ensures that the charges $\mathfrak{P}, \mathfrak{K}$ remain finite in the limit:

$$
\mathfrak{P}=-i \alpha \mathfrak{D}+\mathcal{O}\left(g^{-1}\right), \quad \mathfrak{K}=i \alpha^{-1} \mathfrak{D}+\mathcal{O}\left(g^{-1}\right) .
$$

The fundamental R-matrix becomes trivial in the limit and the first perturbation yields the classical r-matrix,

$$
\mathcal{R}=1 \otimes 1+g^{-1} r+\mathcal{O}\left(g^{-2}\right)
$$

In the classical limit, the Lie brackets of two supercharges (3.3) read

$$
\begin{aligned}
\left\{\mathfrak{Q}^{\alpha}{ }_{b}, \mathfrak{S}^{c}{ }_{\delta}\right\} & =\delta_{b}^{c} \mathfrak{L}^{\alpha}{ }_{\delta}+\delta_{\delta}^{\alpha} \mathfrak{R}^{c}{ }_{b}+\delta_{b}^{c} \delta_{\delta}^{\alpha} \mathfrak{C}, \\
\left\{\mathfrak{Q}^{\alpha}{ }_{b}, \mathfrak{Q}^{\gamma}{ }_{d}\right\} & =-i \alpha \varepsilon^{\alpha \gamma} \varepsilon_{b d} \mathfrak{D}, \\
\left\{\mathfrak{S}^{a}{ }_{\beta}, \mathfrak{S}^{c}{ }_{\delta}\right\} & =i \alpha^{-1} \varepsilon^{a c} \varepsilon_{\beta \delta} \mathfrak{D},
\end{aligned}
$$

i.e. the abelian generator $\mathfrak{D}$ replaces $\mathfrak{P}$ and $\mathfrak{K}$ and becomes part of the Lie algebra. The limit of the coproduct (3.4) yields the cobrackets via (2.15),

$$
\delta\left(\mathfrak{J}_{n}^{A}\right)=\frac{i}{2}[A] \mathfrak{D} \wedge \mathfrak{J}_{n}^{A}+\frac{1}{2} F_{B C}^{A} \sum_{k=0}^{n-1} \mathfrak{J}_{k}^{B} \wedge \mathfrak{J}_{n-1-k}^{C}
$$

The grading for the new generator $\mathfrak{D}$ is obviously trivial $[\mathfrak{D}]=0$.

From these identifications it follows that the evaluation parameter $i u$ for any evaluation representation is fixed in terms of the eigenvalues of $\mathfrak{C}$ and $\mathfrak{D}$,

$$
\mathfrak{J}_{n}^{A} \simeq(i u)^{n} \mathfrak{J}^{A}, \quad u \simeq 2 \mathfrak{C} \mathfrak{D}^{-1}
$$


Fundamental representation. The fundamental representation simplifies somewhat in the classical limit. We choose the following parametrisation [46] for the kinematical variables $x^{ \pm}$:

$$
x^{ \pm}=x \sqrt{1-\frac{1}{4 g^{2}(x-1 / x)^{2}}} \pm \frac{i}{2 g} \frac{x}{x-1 / x}, \quad \gamma=\frac{1}{\sqrt{g}} \tilde{\gamma}
$$

The parameters $\tilde{\gamma}, x, \alpha$ are independent of $g$.

The action of the Lie generators on the 2|2-dimensional representation space spanned by $\left|\phi^{a}\right\rangle$ and $\left|\psi^{\alpha}\right\rangle$ was given in (3.8),(3.9). The limit of the coefficients $a, b, c, d$ are given as follows:

$$
a=\tilde{\gamma}, \quad b=-\frac{i \alpha x}{\tilde{\gamma}\left(x^{2}-1\right)}, \quad c=\frac{i \tilde{\gamma}}{\alpha x}, \quad d=\frac{x^{2}}{\tilde{\gamma}\left(x^{2}-1\right)} .
$$

The eigenvalues of the central charges $\mathfrak{D}$ and $\mathfrak{C}$ read

$$
D=\frac{x}{x^{2}-1}, \quad C=\frac{1}{2} \frac{x^{2}+1}{x^{2}-1} .
$$

Furthermore, the Yangian spectral parameter $u$ is simply given by $u=x+1 / x$, so we immediately confirm (3.24),

$$
u=x+\frac{1}{x}=2 C D^{-1} .
$$

Finally let us mention that for a hermitian representation we should put $\alpha=1, \tilde{\gamma}=\sqrt{x D}$.

Fundamental r-matrix. Let us now take the classical limit of the R-matrix in the fundamental representation using $\mathcal{R}=1 \otimes 1+g^{-1} r$. The resulting representation of the classical r-matrix is given in Table 1, see [26]. ${ }^{6}$ The choice of the phase corresponds to the light cone string S-matrix [38] in (3.17). Instead one could also choose the exact phase factor (3.18) obtained in [34] at the classical level (with a redefinition of length). In that case one would have to add the term $(1 \times 1) r_{0}$ to $r$ with

$$
r_{0} \simeq \frac{i\left(x_{1}-x_{2}\right)\left(x_{1} x_{2}-1\right)}{4\left(x_{1}^{2}-1\right)\left(x_{2}^{2}-1\right)}=\frac{1}{4}\left(i u_{2}-i u_{1}\right) D_{1} D_{2}=\left(1 / i u_{2}-1 / i u_{1}\right) C_{1} C_{2} \text {. }
$$

\section{A Lie Bialgebra for the Classical r-Matrix}

4.1. Moriyama-Torrielli proposal for the classical r-matrix. In [29] the following expression for the classical r-matrix has been proposed:

$$
\begin{aligned}
r_{\mathrm{MT}}=\sum_{m=0}^{\infty} & {\left[+\left(\mathfrak{R}_{m}\right)^{a}{ }_{b} \otimes\left(\tilde{\mathfrak{R}}_{-1-m}\right)^{b}{ }_{a}-\left(\mathfrak{L}_{m}\right)^{\alpha}{ }_{\beta} \otimes\left(\tilde{\mathfrak{L}}_{-1-m}\right)^{\beta}{ }_{\alpha}\right.} \\
& -\left(\mathfrak{R}_{-1-m}\right)^{a}{ }_{b} \otimes\left(\tilde{\mathfrak{R}}_{m}\right)^{b}{ }_{a}+\left(\mathfrak{L}_{-1-m}\right)^{\alpha}{ }_{\beta} \otimes\left(\tilde{\mathfrak{L}}_{m}\right)^{\beta}{ }_{\alpha} \\
& +\left(\mathfrak{Q}_{m}\right)^{\alpha}{ }_{a} \otimes\left(\tilde{\mathfrak{S}}_{-1-m}\right)^{a}{ }_{\alpha}-\left(\mathfrak{S}_{m}\right)^{a}{ }_{\alpha} \otimes\left(\tilde{\mathfrak{Q}}_{-1-m}\right)^{\alpha}{ }_{a} \\
& \left.+\mathfrak{C}_{m} \otimes \tilde{\mathfrak{B}}_{-1-m}+\mathfrak{B}_{m} \otimes \tilde{\mathfrak{C}}_{-1-m}\right] .
\end{aligned}
$$

\footnotetext{
6 One should be able to read off the elements of the diagonalised r-matrix from the integral kernels in [47].
} 
Table 1. The classical (light cone) r-matrix of AdS/CFT

$$
\begin{aligned}
& r\left|\phi^{a} \phi^{b}\right\rangle=\frac{1}{2}\left(A_{12}-B_{12}\right)\left|\phi^{a} \phi^{b}\right\rangle+\frac{1}{2}\left(A_{12}+B_{12}\right)\left|\phi^{b} \phi^{a}\right\rangle+\frac{1}{2} C_{12} \varepsilon^{a b} \varepsilon_{\alpha \beta}\left|\psi^{\alpha} \psi^{\beta}\right\rangle \\
& r\left|\psi^{\alpha} \psi^{\beta}\right\rangle=-\frac{1}{2}\left(D_{12}-E_{12}\right)\left|\psi^{\alpha} \psi^{\beta}\right\rangle-\frac{1}{2}\left(D_{12}+E_{12}\right)\left|\psi^{\beta} \psi^{\alpha}\right\rangle-\frac{1}{2} F_{12} \varepsilon^{\alpha \beta} \varepsilon_{a b}\left|\phi^{a} \phi^{b}\right\rangle \\
& r\left|\phi^{a} \psi^{\beta}\right\rangle=G_{12}\left|\phi^{a} \psi^{\beta}\right\rangle+H_{12}\left|\psi^{\beta} \phi^{a}\right\rangle \\
& r\left|\psi^{\alpha} \phi^{b}\right\rangle=K_{12}\left|\phi^{b} \psi^{\alpha}\right\rangle+L_{12}\left|\psi^{\alpha} \phi^{b}\right\rangle \\
& \frac{1}{2}\left(A_{12}+B_{12}\right)=\frac{1}{i u_{1}-i u_{2}} \\
& \frac{1}{2}\left(A_{12}-B_{12}\right)=\frac{\left(x_{1}-x_{2}\right)^{2}\left(x_{1} x_{2}+1\right)^{2}}{4 x_{1} x_{2}\left(x_{1}^{2}-1\right)\left(x_{2}^{2}-1\right)\left(i u_{1}-i u_{2}\right)}=\frac{+\frac{1}{2}+\frac{1}{4} D_{1} D_{2}^{-1}+\frac{1}{4} D_{1}^{-1} D_{2}}{i u_{1}-i u_{2}} \\
& \frac{1}{2} C_{12}=\frac{i \tilde{\gamma}_{1} \tilde{\gamma}_{2}\left(x_{1}-x_{2}\right)}{\alpha x_{1} x_{2}\left(i u_{1}-i u_{2}\right)}=\frac{a_{1} c_{2}-c_{1} a_{2}}{i u_{1}-i u_{2}} \\
& -\frac{1}{2}\left(D_{12}+E_{12}\right)=-\frac{1}{i u_{1}-i u_{2}} \\
& -\frac{1}{2}\left(D_{12}-E_{12}\right)=-\frac{\left(x_{1}-x_{2}\right)^{2}\left(x_{1} x_{2}+1\right)^{2}}{4 x_{1} x_{2}\left(x_{1}^{2}-1\right)\left(x_{2}^{2}-1\right)\left(i u_{1}-i u_{2}\right)}=\frac{-\frac{1}{2}-\frac{1}{4} D_{1} D_{2}^{-1}-\frac{1}{4} D_{1}^{-1} D_{2}}{i u_{1}-i u_{2}} \\
& -\frac{1}{2} F_{12}=-\frac{i \alpha x_{1} x_{2}\left(x_{1}-x_{2}\right)}{\tilde{\gamma}_{1} \tilde{\gamma}_{2}\left(x_{1}^{2}-1\right)\left(x_{2}^{2}-1\right)\left(i u_{1}-i u_{2}\right)}=\frac{d_{1} b_{2}-b_{1} d_{2}}{i u_{1}-i u_{2}} \\
& G_{12}=\frac{\left(x_{1}^{2}-x_{2}^{2}\right)\left(x_{1}^{2} x_{2}^{2}-1\right)}{4 x_{1} x_{2}\left(x_{1}^{2}-1\right)\left(x_{2}^{2}-1\right)\left(i u_{1}-i u_{2}\right)}=\frac{-\frac{1}{4} D_{1} D_{2}^{-1}+\frac{1}{4} D_{1}^{-1} D_{2}}{i u_{1}-i u_{2}} \\
& H_{12}=\frac{\tilde{\gamma}_{1} x_{2}\left(x_{1} x_{2}-1\right)}{\tilde{\gamma}_{2} x_{1}\left(x_{2}^{2}-1\right)\left(i u_{1}-i u_{2}\right)}=\frac{a_{1} d_{2}-c_{1} b_{2}}{i u_{1}-i u_{2}} \\
& K_{12}=\frac{\tilde{\gamma}_{2} x_{1}\left(x_{1} x_{2}-1\right)}{\tilde{\gamma}_{1} x_{2}\left(x_{1}^{2}-1\right)\left(i u_{1}-i u_{2}\right)}=\frac{d_{1} a_{2}-b_{1} c_{2}}{i u_{1}-i u_{2}} \\
& L_{12}=-\frac{\left(x_{1}^{2}-x_{2}^{2}\right)\left(x_{1}^{2} x_{2}^{2}-1\right)}{4 x_{1} x_{2}\left(x_{1}^{2}-1\right)\left(x_{2}^{2}-1\right)\left(i u_{1}-i u_{2}\right)}=\frac{+\frac{1}{4} D_{1} D_{2}^{-1}-\frac{1}{4} D_{1}^{-1} D_{2}}{i u_{1}-i u_{2}}
\end{aligned}
$$

Formally, it looks similar to the standard $\mathfrak{u}(2 \mid 2)$ classical r-matrix in (2.20). The only difference is that for the $\mathfrak{s u}(2) \times \mathfrak{s u}(2)$ generators there are additional terms with inverted level numbers in the second line.

To recover the above fundamental $r$-matrix in Table 1 , a representation quite different from the standard evaluation representation was used:

$$
\begin{aligned}
&\left(\mathfrak{Q}_{m}\right)^{\alpha}{ }_{b} \simeq\left(\tilde{\mathfrak{Q}}_{m}\right)^{\alpha}{ }_{b} \simeq \mathfrak{Q}^{\alpha}{ }_{b}\left(x^{m} \Pi_{\mathrm{b}}+x^{-m} \Pi_{\mathrm{f}}\right), \\
&\left(\mathfrak{S}_{m}\right)^{a}{ }_{\beta} \simeq\left(\tilde{\mathfrak{S}}_{m}\right)^{a}{ }_{\beta} \simeq \mathfrak{S}^{a}{ }_{\beta}\left(x^{m} \Pi_{\mathrm{f}}+x^{-m} \Pi_{\mathrm{b}}\right), \\
&\left(\mathfrak{R}_{m}\right)^{a}{ }_{b} \simeq \mathfrak{R}^{a}{ }_{b} \frac{x^{m+1}-x^{-m-1}}{x-x^{-1}}, \\
&\left(\tilde{\mathfrak{R}}_{m}\right)^{a}{ }_{b} \simeq-\mathfrak{R}^{a}{ }_{b} \frac{x^{m-1}-x^{-m+1}}{x-x^{-1}}, \\
&\left(\mathfrak{L}_{m}\right)^{\alpha}{ }_{\beta} \simeq \mathfrak{L}^{\alpha}{ }_{\beta} \frac{x^{m+1}-x^{-m-1}}{x-x^{-1}},
\end{aligned}
$$




$$
\begin{aligned}
\left(\tilde{\mathfrak{L}}_{m}\right)^{\alpha}{ }_{\beta} & \simeq-\mathfrak{L}^{\alpha}{ }_{\beta} \frac{x^{m-1}-x^{-m+1}}{x-x^{-1}}, \\
\mathfrak{C}_{m} \simeq \tilde{\mathfrak{C}}_{m} & \simeq \frac{1}{2} \frac{x^{m+1}+x^{-m-1}}{x-x^{-1}}, \\
\mathfrak{B}_{m} \simeq \tilde{\mathfrak{B}}_{m} & \simeq \frac{1}{2}\left(x^{m}-x^{-m}\right) .
\end{aligned}
$$

This representation is quite unusual since it does not treat the different generators on an equal footing and since it makes a distinction between bosons and fermions by means of the projection operators $\Pi_{b, f}$. The argument of the authors of [29] not to use the standard evaluation representation $\mathfrak{J}_{n}=x^{n} \mathfrak{J}$ was due to the fact that that this would lead to a classical r-matrix with poles only in $x_{1}=x_{2}$ and none at $x_{1}=1 / x_{2}$, in agreement with Table 1. Furthermore, the proposed Lie brackets look quite complicated; they are not of a standard loop algebra form, and we shall not reproduce them here. We do not know whether the brackets obey the Jacobi identities and whether the r-matrix satisfies the CYBE using these brackets; no statement was made in [29].

In contrast, it was shown in [17] that the $\mathfrak{p s u}(2 \mid 2) \ltimes \mathbb{R}^{3}$ fundamental R-matrix is invariant under Yangian generators for an ordinary evaluation representation with evaluation parameter $i u$ with $u=x+1 / x$. A reason for this superficial mismatch was proposed in [29]: The work [17] was formulated in Drinfeld's first realisation of the Yangian, and the work [29] was formulated in the second. In principle there might be a non-trivial map between the two realisations which would make the two representations equivalent.

We should note that the procedure applied in [29] is not necessarily unique. There may be several representations leading to the same fundamental r-matrix in Table 1 upon inserting into the above classical $r$-matrix because often several terms of the classical r-matrix contribute to a single matrix element of the fundamental r-matrix. One needs to make a choice of how to distribute the individual terms to these contributions from the classical r-matrix. Apparently the choice of a symmetric distribution was made in [29] which led to the above representation. Furthermore, it was admitted in [29] that the resulting algebra is not unique.

Here we note that a loop variable $i u$ with $u=x+1 / x$ automatically leads to poles at $x_{1}=x_{2}$ and $x_{1}=1 / x_{2}$ as required for the fundamental $r$-matrix. Instead of producing just the right poles in each term, one might in this way attempt to cancel the wrong poles. As the determination of the representation is not unique, this can indeed lead to the same fundamental $r$-matrix using the above (or a similar) classical r-matrix. In the remainder of this section we shall make an alternative proposal for a normal evaluation representation based on $i u$, a consistent Lie algebra and a classical r-matrix obeying the CYBE. This leads to a direct analog of the Yangian considered in [17]. At this point we cannot say whether the proposal of [29] is consistent with ours and merely represents a very different choice of basis. A change of basis can indeed lead to superficially quite different algebras as the example in Sect. 5.1 shows. In any case, we find our proposal more natural and it is probably easier to deal with because it employs standard evaluation representations and an (almost) standard loop algebra.

4.2. Observation. The standard form for the classical r-matrix (2.20) makes use of the quadratic Casimir. Unfortunately, it does not exist for our algebra $\mathfrak{h}$ because the $\mathfrak{s l}(2)$ automorphisms would be required to complement the central charges, see Sect. 5.2. 
Nevertheless the quadratic Casimir operator for $\mathfrak{p s u}(2 \mid 2)$ can be written within $\mathfrak{h}$,

$$
\mathcal{T}=\frac{1}{2} \mathfrak{R}^{c}{ }_{d} \mathfrak{R}^{d}{ }_{c}-\frac{1}{2} \mathfrak{L}^{\gamma}{ }_{\delta} \mathfrak{L}^{\delta}{ }_{\gamma}+\frac{1}{2} \mathfrak{Q}^{\gamma}{ }_{d} \mathfrak{S}^{d}{ }_{\gamma}-\frac{1}{2} \mathfrak{S}^{c}{ }_{\delta} \mathfrak{Q}^{\delta}{ }_{c} .
$$

The corresponding two-site operator reads

$$
\mathcal{T}_{12}=\mathfrak{R}^{c}{ }_{d} \otimes \mathfrak{R}^{d}{ }_{c}-\mathfrak{L}^{\gamma}{ }_{\delta} \otimes \mathfrak{L}^{\delta}{ }_{\gamma}+\mathfrak{Q}^{\gamma}{ }_{d} \otimes \mathfrak{S}^{d}{ }_{\gamma}-\mathfrak{S}^{c}{ }_{\delta} \otimes \mathfrak{Q}^{\delta}{ }_{c} .
$$

Here we make the crucial observation that all the off-diagonal elements of the r-matrix in Table 1 are generated by the operator $\mathcal{T}_{12} /\left(i u_{1}-i u_{2}\right)$. To make this statement more transparent, we have written the coefficients in Table 1 in an alternative form using the coefficients $a, b, c, d$ (3.26) which determine the action of supercharges. The diagonal elements, however, are not reproduced correctly. Nevertheless the remainder takes a peculiar form in which two signs only depend on whether the state the r-matrix acts upon consists of bosons or fermions. Formally, we can achieve full agreement with the fundamental r-matrix by the following expression:

$$
r_{12}=\frac{\mathcal{T}_{12}-\mathcal{T} \mathfrak{D}^{-1} \otimes \mathfrak{D}-\mathfrak{D} \otimes \mathcal{T} \mathfrak{D}^{-1}}{i u_{1}-i u_{2}} .
$$

However, this is not an element of $\mathfrak{h} \otimes \mathfrak{h}$ but rather of its enveloping algebra. Furthermore, the element $\mathfrak{D}$ is strictly speaking not invertible. That means that formally the expression (4.5) may be used to compute the r-matrix in evaluation representations, but it is not a universal r-matrix. Before we continue, let us rewrite the r-matrix in a slightly different manner:

$$
\begin{aligned}
r_{12} & =\frac{\mathcal{T}_{12}-\left(i u_{1} / i u_{2}\right) \mathcal{T} \mathfrak{C}^{-1} \otimes \mathfrak{C}-\left(i u_{2} / i u_{1}\right) \mathfrak{C} \otimes \mathcal{T} \mathfrak{C}^{-1}}{i u_{1}-i u_{2}} \\
& =\frac{\mathcal{T}_{12}-\mathcal{T} \mathfrak{C}^{-1} \otimes \mathfrak{C}-\mathfrak{C} \otimes \mathcal{T} \mathfrak{C}^{-1}}{i u_{1}-i u_{2}}-\frac{\mathcal{T} \mathfrak{C}^{-1} \otimes \mathfrak{C}}{i u_{2}}+\frac{\mathfrak{C} \otimes \mathcal{T} \mathfrak{C}^{-1}}{i u_{1}}
\end{aligned}
$$

Clearly we have not gained anything by this transformation, but this will be a more convenient starting point for our further analysis.

4.3. A deformation of the $\mathfrak{u}(2 \mid 2)$ loop algebra. To accommodate the r-matrix in a Lie bialgebra it should consist of bilinear terms in the generators only. Instead of $\mathcal{T} \mathfrak{C}^{-1}$ we should have a single Lie generator $\mathfrak{B}$. Let us therefore examine the commutators of this combination and see if we can interpret them as Lie brackets

$$
\begin{aligned}
& {\left[\mathcal{T} \mathfrak{C}^{-1}, \mathfrak{Q}^{\alpha}{ }_{b}\right]=+\mathfrak{Q}^{\alpha}{ }_{b}+i \alpha \varepsilon^{\alpha \gamma} \varepsilon_{b d} \mathfrak{D C}^{-1} \mathfrak{S}^{d}{ }_{\gamma},} \\
& {\left[\mathcal{T} \mathfrak{C}^{-1}, \mathfrak{S}_{\beta}^{a}{ }_{\beta}\right]=-\mathfrak{S}^{a}{ }_{\beta}+i \alpha^{-1} \varepsilon^{a c} \varepsilon_{\beta \delta} \mathfrak{D} \mathfrak{C}^{-1} \mathfrak{Q}^{\delta}{ }_{c} .}
\end{aligned}
$$

The resulting linear terms are clearly okay. For the cubic terms we note that $u=2 C D^{-1}$, which means that we may interpret the combination $\mathfrak{D C}^{-1}$ as a shift in the level of a loop algebra generator. If we introduce $\mathfrak{B}$ such that its brackets coincide with commutators of $\mathcal{T} \mathfrak{C}^{-1}$, the loop algebra becomes

$$
\begin{aligned}
& {\left[\mathfrak{B}_{m},\left(\mathfrak{Q}_{n}\right)^{\alpha}{ }_{b}\right]=+\left(\mathfrak{Q}_{m+n}\right)^{\alpha}{ }_{b}-2 \alpha \beta \varepsilon^{\alpha \gamma} \varepsilon_{b d}\left(\mathfrak{S}_{m+n-1}\right)^{d}{ }_{\gamma},} \\
& {\left[\mathfrak{B}_{m},\left(\mathfrak{S}_{n}\right)^{a}{ }_{\beta}\right]=-\left(\mathfrak{S}_{m+n}\right)^{a}{ }_{\beta}-2 \alpha^{-1} \beta \varepsilon^{a c} \varepsilon_{\beta \delta}\left(\mathfrak{Q}_{m+n-1}\right)^{\delta} .}
\end{aligned}
$$


Here we have introduced a parameter $\beta$ which in our case equals $\beta=1 .^{7}$ In fact these relations are very reminiscent of the automorphism in $\mathfrak{u}(2 \mid 2)$. The deformation parameter $\beta$ in fact interpolates between the standard $\mathfrak{u}(2 \mid 2)$ loop algebra, which we get for $\beta=0$, and our algebra. ${ }^{8}$ The remaining brackets of $\mathfrak{B}$ should be trivial

$$
\left[\mathfrak{B}_{m}, \mathfrak{C}_{n}\right]=\left[\mathfrak{B}_{m},\left(\mathfrak{R}_{n}\right)^{a}{ }_{b}\right]=\left[\mathfrak{B}_{m},\left(\mathfrak{L}_{n}\right)^{\alpha}{ }_{\beta}\right]=0 .
$$

Finally, because we have $u=2 C D^{-1}$, we may identify $\mathfrak{D}_{n}=2 i \mathfrak{C}_{n-1}$ and obtain for the brackets of supercharges

$$
\begin{aligned}
\left\{\left(\mathfrak{Q}_{m}\right)^{\alpha}{ }_{b},\left(\mathfrak{S}_{n}\right)^{c}{ }_{\delta}\right\} & =\delta_{b}^{c}\left(\mathfrak{L}_{m+n}\right)^{\alpha}{ }_{\delta}+\delta_{\delta}^{\alpha}\left(\mathfrak{R}_{m+n}\right)^{c}{ }_{b}+\delta_{b}^{c} \delta_{\delta}^{\alpha}\left(\mathfrak{C}_{m+n}\right), \\
\left\{\left(\mathfrak{Q}_{m}\right)^{\alpha}{ }_{b},\left(\mathfrak{Q}_{n}\right)^{\gamma}{ }_{d}\right\} & =2 \alpha \beta \varepsilon^{\alpha \gamma} \varepsilon_{b d} \mathfrak{C}_{m+n-1}, \\
\left\{\left(\mathfrak{S}_{m}\right)^{a}{ }_{\beta},\left(\mathfrak{S}_{n}\right)^{c}{ }_{\delta}\right\} & =-2 \alpha^{-1} \beta \varepsilon^{a c} \varepsilon_{\beta \delta} \mathfrak{C}_{m+n-1} .
\end{aligned}
$$

The brackets of $\mathfrak{s u}(2) \times \mathfrak{s u}(2)$ are undeformed and given in (3.2) (supplemented with additive levels of the loop algebra). It is not difficult to confirm that these brackets obey the Jacobi identity for arbitrary $\alpha, \beta$ and therefore they define a family of Lie algebras. In fact, the algebra can be embedded into the regular $\mathfrak{u}(2 \mid 2)$ loop algebra as we shall see below in Sect. 5.1.

The action of $\mathfrak{B}$ on the fundamental representation for $\beta=1$ should be equal to $\mathcal{T} \mathfrak{C}^{-1}$ which yields

$$
\mathfrak{B}\left|\phi^{a}\right\rangle=-\frac{1}{4 C}\left|\phi^{a}\right\rangle, \quad \mathfrak{B}\left|\psi^{\alpha}\right\rangle=+\frac{1}{4 C}\left|\psi^{\alpha}\right\rangle .
$$

4.4. Classical r-matrix and cobrackets. We can now write down a classical r-matrix for our Lie algebra by substituting $\mathfrak{B}$ for $\mathcal{T} \mathfrak{C}^{-1}$ in (4.6),

$$
r_{12}=\frac{\mathcal{T}_{12}-\mathfrak{B} \otimes \mathfrak{C}-\mathfrak{C} \otimes \mathfrak{B}}{i u_{1}-i u_{2}}-\frac{\mathfrak{B} \otimes \mathfrak{C}}{i u_{2}}+\frac{\mathfrak{C} \otimes \mathfrak{B}}{i u_{1}} .
$$

This expression assumes evaluation representations, but we can reexpress it in full generality using loop algebra generators

$$
r=r_{\mathfrak{p s u}(2 \mid 2)}-\sum_{m=-1}^{\infty} \mathfrak{B}_{-1-m} \otimes \mathfrak{C}_{m}-\sum_{m=+1}^{\infty} \mathfrak{C}_{-1-m} \otimes \mathfrak{B}_{m}
$$

with the classical r-matrix $r_{\mathfrak{p s u}(2 \mid 2)}$ for $\mathfrak{p s u}(2 \mid 2)$,

$$
\begin{aligned}
r_{\mathfrak{p s u}(2 \mid 2)=} & +\sum_{m=0}^{\infty}\left(\mathfrak{R}_{-1-m}\right)^{c}{ }_{d} \otimes\left(\mathfrak{R}_{m}\right)^{d}{ }_{c}-\sum_{m=0}^{\infty}\left(\mathfrak{L}_{-1-m}\right)^{\gamma_{\delta}} \otimes\left(\mathfrak{L}_{m}\right)^{\delta}{ }_{\gamma} \\
& +\sum_{m=0}^{\infty}\left(\mathfrak{Q}_{-1-m}\right)^{\gamma_{d}} \otimes\left(\mathfrak{S}_{m}\right)^{d}{ }_{\gamma}-\sum_{m=0}^{\infty}\left(\mathfrak{S}_{-1-m}\right)^{c}{ }_{\delta} \otimes\left(\mathfrak{Q}_{m}\right)^{\delta}{ }_{c} .
\end{aligned}
$$

\footnotetext{
${ }^{7}$ In fact one may keep the parameter $\beta$ arbitrary if one inserts it into the relations (3.6) as well. Essentially $\beta$ corresponds to a rescaling of $g$.

${ }^{8}$ Note that we also have $\mathfrak{u}(2 \mid 2)$ symmetry in the alternative limit discussed in Sect. 6, where we provide further details.
} 
This expression is almost the standard form for $\mathfrak{u}(2 \mid 2)\left[i u,(i u)^{-1}\right]$, but note that the lower bound on the sum for the $\mathfrak{B}-\mathfrak{C}$ terms is shifted by \pm 1 due to the extra terms in (4.12). To motivate the extra term $\mathfrak{C}_{-1} \wedge \mathfrak{B}_{0}$ recall that the coproduct (3.4) is not the ordinary Yangian coproduct but contains an additional braiding factor. For undeformed $\mathfrak{u}(2 \mid 2)\left[i u,(i u)^{-1}\right]$ this braided coproduct can easily be obtained from the standard coproduct via a Reshetikhin twist transformation [48]

$$
\Delta(\mathfrak{J})=\mathcal{F} \Delta_{0}(\mathfrak{J}) \mathcal{F}^{-1}, \quad \mathcal{R}=\mathcal{P}(\mathcal{F}) \mathcal{R}_{0} \mathcal{F}^{-1},
$$

with $\mathcal{F}=\exp \left(-g^{-1} \mathfrak{C}_{-1} \otimes \mathfrak{B}_{0}\right)$. The requirements for the transformation in [48] are satisfied because the coproducts of the Cartan elements $\mathfrak{C}_{-1}$ and $\mathfrak{B}_{0}$ are both trivial. The twist $\mathcal{F}=1 \otimes 1+g^{-1} f+\mathcal{O}\left(g^{-2}\right)$ will contribute to the classical r-matrix by the term $-f+\mathcal{P}(f)$. Indeed, $\mathfrak{C}_{-1} \wedge \mathfrak{B}_{0}=-f+\mathcal{P}(f)$ is the classical contribution from the twist.

It is also straightforward to include the AFS phase (3.29) by adding to (4.13),

$$
r_{0}=-\mathfrak{C}_{-1} \wedge \mathfrak{C}_{0}
$$

Note that curiously one can combine the extra term discussed above with the phase into $\mathfrak{C}_{-1} \wedge\left(\mathfrak{B}_{0}-\mathfrak{C}_{0}\right)$. This shift clearly has no impact on any of the relevant properties of classical r-matrices because the $\mathfrak{C}_{n}$ are central elements of the algebra. In fact, one can incorporate arbitrary phase because terms of the sort $\mathfrak{C}_{m} \wedge \mathfrak{C}_{n}$ do not modify any of the relevant properties of classical r-matrices. ${ }^{9}$ With these terms one can represent an arbitrary antisymmetric function of the two variables $u_{1}$ and $u_{2}$ by

$$
r_{0}=\sum_{m, n=-\infty}^{\infty} c_{m, n} \mathfrak{C}_{m} \wedge \mathfrak{C}_{n}
$$

with antisymmetric coefficients $c_{m, n}$. Note that these contributions can also be viewed as a Reshetikhin twist similarly to the above discussion.

We can now determine the cobrackets from the r-matrix via the standard relation (2.14); the results are summarised in Table 2. These expressions agree exactly with the expected cobrackets for centrally extended $\mathfrak{p s u}(2 \mid 2)$ in (3.23) when we set the deformation parameter $\beta=1$. We also see that cobracket $\delta\left(\mathfrak{B}_{1}\right)=\mathfrak{Q}^{\alpha}{ }_{b} \wedge \mathfrak{S}^{b}{ }_{\alpha}$, which is not part of centrally extended $\mathfrak{s u}(2 \mid 2)$, is consistent with the coproduct of the combination $2 i \mathcal{T} \mathfrak{D}^{-1}$ in the Hopf algebra.

Finally, we should prove the CYBE $[[r, r]]=0$. A convenient method is to split up the computation into three parts. In the first part we shall set $\beta=0$ and also adjust all lower bounds of the sums in (4.13) to $m=0$. Then we have the standard rational r-matrix for the algebra $\mathrm{u}(2 \mid 2)\left[i u,(i u)^{-1}\right]$ which is known to satisfy the CYBE.

Secondly, we have omitted the term $\mathfrak{C}_{-1} \wedge \mathfrak{B}_{0}$ by adjusting the summation bounds. As discussed above, this term originates from a Reshetikhin twist and thus preserves the CYBE when $\beta=0$. More explicitly, we add a term of the sort

$$
r^{\prime}=r+\mathfrak{J} \wedge \mathfrak{J}^{\prime}
$$

This changes the commutators in the CYBE to

$$
\left[\left[r^{\prime}, r^{\prime}\right]\right]=[[r, r]]+\mathfrak{J} \wedge \wedge\left[\mathfrak{J}^{\prime}, r\right]-\mathfrak{J}^{\prime} \wedge \wedge[\mathfrak{J}, r]+\mathfrak{J} \wedge \mathfrak{J}^{\prime} \wedge\left[\mathfrak{J}, \mathfrak{J}^{\prime}\right],
$$

\footnotetext{
${ }^{9}$ In a lift to the quantum theory, however, the Hopf coproduct of central charges is not necessarily trivial and therefore the set of possible twists will be reduced by demanding quasi-triangularity.
} 
Table 2. Cobrackets of the Lie bialgebra generators

$$
\begin{aligned}
& \delta\left(\mathfrak{C}_{n}\right)=0 \\
& \delta\left(\mathfrak{B}_{n}\right)=+\sum_{k=0}^{n-1}\left(\mathfrak{Q}_{k}\right)^{\alpha}{ }_{b} \wedge\left(\mathfrak{S}_{n-1-k}\right)^{b}{ }_{\alpha} \\
& +\sum_{k=1}^{n-1} \alpha^{-1} \beta \varepsilon^{b d} \varepsilon_{\alpha \gamma}\left(\mathfrak{Q}_{k-1}\right)^{\alpha}{ }_{b} \wedge\left(\mathfrak{Q}_{n-1-k}\right)^{\gamma} d \\
& -\sum_{k=1}^{n-1} \alpha \beta \varepsilon^{\beta \delta} \varepsilon_{a c}\left(\mathfrak{S}_{k-1}\right)^{a}{ }_{\beta} \wedge\left(\mathfrak{S}_{n-1-k}\right)_{\delta}^{c} \\
& \delta\left(\Re_{n}\right)^{a}{ }_{b}=+\sum_{k=0}^{n-1}\left(\Re_{k}\right)^{a}{ }_{c} \wedge\left(\Re_{n-1-k}\right)^{c}{ }_{b} \\
& -\sum_{k=0}^{n-1}\left[\left(\mathfrak{S}_{k}\right)^{a}{ }_{\gamma} \wedge\left(\mathfrak{Q}_{n-1-k}\right)^{\gamma}{ }_{b}-\frac{1}{2} \delta_{b}^{a}\left(\mathfrak{S}_{k}\right)^{d}{ }_{\gamma} \wedge\left(\mathfrak{Q}_{n-1-k}\right)^{\gamma} d\right] \\
& \delta\left(\mathfrak{L}_{n}\right)^{\alpha}{ }_{\beta}=-\sum_{k=0}^{n-1}\left(\mathfrak{L}_{k}\right)^{\alpha} \gamma \wedge\left(\mathfrak{L}_{n-1-k}\right)^{\gamma} \beta \\
& +\sum_{k=0}^{n-1}\left[\left(\mathfrak{Q}_{k}\right)^{\alpha}{ }_{c} \wedge\left(\mathfrak{S}_{n-1-k}\right)^{c}{ }_{\beta}-\frac{1}{2} \delta_{\beta}^{\alpha}\left(\mathfrak{Q}_{k}\right)^{\delta}{ }_{c} \wedge\left(\mathfrak{S}_{n-1-k}\right)^{c} \delta\right] \\
& \delta\left(\mathfrak{Q}_{n}\right)^{\alpha}{ }_{b}=-\sum_{k=0}^{n-1}\left(\mathfrak{L}_{k}\right)^{\alpha}{ }_{\gamma} \wedge\left(\mathfrak{Q}_{n-1-k}\right)^{\gamma}{ }_{b}-\sum_{k=0}^{n-1}\left(\mathfrak{R}_{k}\right)^{c}{ }_{b} \wedge\left(\mathfrak{Q}_{n-1-k}\right)^{\alpha}{ }_{c} \\
& -\sum_{k=0}^{n} \mathfrak{C}_{k-1} \wedge\left(\mathfrak{Q}_{n-k}\right)^{\alpha}{ }_{b}+\sum_{k=0}^{n-1} 2 \alpha \beta \varepsilon^{\alpha \gamma} \varepsilon_{b d} \mathfrak{C}_{k-1} \wedge\left(\mathfrak{S}_{n-1-k}\right)^{d} \gamma \\
& \delta\left(\mathfrak{S}_{n}\right)^{a}{ }_{\beta}=+\sum_{k=0}^{n-1}\left(\mathfrak{R}_{k}\right)^{a}{ }_{c} \wedge\left(\mathfrak{S}_{n-1-k}\right)^{c}{ }_{\beta}+\sum_{k=0}^{n-1}\left(\mathfrak{L}_{k}\right)^{\gamma}{ }_{\beta} \wedge\left(\mathfrak{S}_{n-1-k}\right)^{a} \gamma \\
& +\sum_{k=0}^{n} \mathfrak{C}_{k-1} \wedge\left(\mathfrak{S}_{n-k}\right)^{a}{ }_{\beta}+\sum_{k=0}^{n-1} 2 \alpha^{-1} \beta \varepsilon^{a c} \varepsilon_{\beta \delta} \mathfrak{C}_{k-1} \wedge\left(\mathfrak{Q}_{n-1-k}\right)^{\delta_{c}}
\end{aligned}
$$

where we define the double-wedge as

$$
\mathfrak{J}^{A} \wedge \wedge\left(\mathfrak{J}^{B} \otimes \mathfrak{J}^{C}\right):=\mathfrak{J}^{A} \otimes \mathfrak{J}^{B} \otimes \mathfrak{J}^{C}-\mathfrak{J}^{B} \otimes \mathfrak{J}^{A} \otimes \mathfrak{J}^{C}+\mathfrak{J}^{B} \otimes \mathfrak{J}^{C} \otimes \mathfrak{J}^{A}
$$

For $\mathfrak{J}=\mathfrak{C}_{-1}$ and $\mathfrak{J}^{\prime}=\mathfrak{B}_{0}$ all the additional terms vanish because $\mathfrak{C}_{-1}$ and $\mathfrak{B}_{0}$ are both Cartan elements and thus obey $\left[\mathfrak{B}_{0}, r\right]=\left[\mathfrak{C}_{-1}, r\right]=\left[\mathfrak{C}_{-1}, \mathfrak{B}_{0}\right]=0$.

It remains to confirm the CYBE for all contributions proportional to the deformation $\beta$. These originate from the brackets $[\mathfrak{B}, \mathfrak{Q}],[\mathfrak{B}, \mathfrak{S}]$, as well as $\{\mathfrak{Q}, \mathfrak{Q}\}$ and $\{\mathfrak{S}, \mathfrak{S}\}$. It is relatively easy to confirm that these terms cancel. Here the modification of the summation bounds in (4.13) is crucial; without it some terms would remain. ${ }^{10}$

10 This is in agreement with the fact that $\mathfrak{C}_{-1} \wedge \mathfrak{B}_{0}$ does not correspond to a Reshetikhin twist of the deformed algebra because $\left[\mathfrak{B}_{0}, r\right]=\delta\left(\mathfrak{B}_{0}\right) \neq 0$ and thus $\left[\left[r^{\prime}, r^{\prime}\right]\right] \neq 0$ according to (4.19). 
4.5. The deformed loop algebra as a classical double. In this section we want to show that the deformed loop algebra including the r-matrix (4.5) can be obtained via a classical double construction. If a bialgebra can be written as a double it is automatically quasitriangular. In general, the classical double $\mathrm{D}(\mathfrak{g})$ of a Lie bialgebra $\mathfrak{g}$ is the vector space

$$
\mathrm{D}(\mathfrak{g})=\mathfrak{g} \oplus \mathfrak{g}^{*} .
$$

The Lie brackets of $\mathrm{D}(\mathfrak{g})$ read

$$
\left[\mathfrak{J}^{A}, \mathfrak{J}^{B}\right]=F_{C}^{A B} \mathfrak{J}^{C}, \quad\left[\mathfrak{J}_{A}, \mathfrak{J}_{B}\right]=\tilde{F}_{A B}^{C} \mathfrak{J}_{C}, \quad\left[\mathfrak{J}^{A}, \mathfrak{J}_{B}\right]=\tilde{F}_{B C}^{A} \mathfrak{J}^{C}-F_{B}^{A C} \mathfrak{J}_{C},
$$

with $\mathfrak{J}^{A}$ forming a basis of $\mathfrak{g}$ and $\mathfrak{J}_{A}$ being the corresponding dual basis of $\mathfrak{g}^{*}$. The coalgebra structure of $\mathrm{D}(\mathfrak{g})$ is simply given by the canonical r-matrix

$$
r=\mathfrak{J}^{A} \otimes \mathfrak{J}_{A} .
$$

One can convince oneself that the induced cobracket reads

$$
\delta\left(\mathfrak{J}^{A}\right)=\tilde{F}_{B C}^{A} \mathfrak{J}^{B} \otimes \mathfrak{J}^{C}, \quad \delta\left(\mathfrak{J}_{A}\right)=-F_{A}^{B C} \mathfrak{J}_{B} \otimes \mathfrak{J}_{C} .
$$

and that the bialgebra $\mathrm{D}(\mathfrak{g})$ is quasi-triangular.

In the standard loop algebra $\mathfrak{g}\left[u, u^{-1}\right]$ of a Lie algebra $\mathfrak{g}$ with non-degenerate invariant bilinear form $C_{A B}$ we may take the decomposition into the subalgebra $\mathfrak{g}^{+}=\mathfrak{g}[u]$ consisting of generators with non-negative powers in $u$ and the subalgebra $\mathfrak{g}^{-}=u^{-1} \mathfrak{g}\left[u^{-1}\right]$ consisting of generators of negative powers in $u$. Then we indeed have ${ }^{11}\left(\mathfrak{g}^{+}\right)^{*}=\mathfrak{g}^{-}$ and $\mathrm{D}\left(\mathfrak{g}^{+}\right)=\mathfrak{g}^{+} \oplus \mathfrak{g}^{-}=\mathfrak{g}\left[u, u^{-1}\right]$. A dual pairing between the two subalgebras is given by

$$
\left(\mathfrak{J}_{n}^{A}, \mathfrak{J}_{m}^{B}\right)=-\delta_{n,-m-1} C^{A B}
$$

with $C^{A B}$ the Cartan-Killing matrix of $\mathfrak{g}$. It defines a consistent cobracket on $\mathfrak{g}^{+}$from the bracket of $\mathfrak{g}^{-}$. The induced classical r-matrix in (4.23) then reads

$$
r=-\sum_{n=0}^{\infty} C_{A B} \mathfrak{J}_{n}^{A} \otimes \mathfrak{J}_{-n-1}^{B},
$$

which is precisely one of the asymmetric r-matrices of (2.20). The other one is obtained by exchanging $\mathfrak{g}^{+}$and $\mathfrak{g}^{-}$, i.e. considering the double of $\mathfrak{g}^{-}$.

The case of our deformed $\mathfrak{u}(2 \mid 2)$ works almost in the same way as for generic loop algebras. Due to the deformed commutation relations of the automorphisms $\mathfrak{B}_{n}$ and the identification of the loop variable with the central charges we should actually set

$$
\begin{aligned}
\mathfrak{g}^{+} & =\left\langle\mathfrak{R}_{n}, \mathfrak{L}_{n}, \mathfrak{Q}_{n}, \mathfrak{S}_{n}, \mathfrak{C}_{n-1}, \mathfrak{B}_{n+1}\right\rangle_{n \geq 0}, \\
\mathfrak{g}^{-} & =\left\langle\mathfrak{R}_{-1-n}, \mathfrak{L}_{-1-n}, \mathfrak{Q}_{-1-n}, \mathfrak{S}_{-1-n}, \mathfrak{C}_{-2-n}, \mathfrak{B}_{-n}\right\rangle_{n \geq 0} .
\end{aligned}
$$

With this assignment $\mathfrak{g}^{+}$and $\mathfrak{g}^{-}$are indeed sub-bialgebras of $\mathfrak{g}$, i.e. the brackets (4.8), (4.10) and the cobrackets (Table 2) close on $\mathfrak{g}^{+}$and $\mathfrak{g}^{-}$, respectively; the level shift for $\mathfrak{B}_{n}$ and $\mathfrak{C}_{n}$ is crucial for this property. Furthermore, the r-matrix (4.13),(4.14) corresponds to a dual pairing between $\mathfrak{g}^{+}$and $\mathfrak{g}^{-}$. These properties suffice to show that the deformed $\mathfrak{u}(2 \mid 2)\left[u, u^{-1}\right]$ loop algebra is the double $\mathrm{D}\left(\mathfrak{g}^{-}\right)$.

\footnotetext{
11 Strictly speaking, we should pair polynomials $\mathfrak{g}[u]$ with formal power series $u^{-1} \mathfrak{g}\left[\left[u^{-1}\right]\right]$, resulting in the double $\mathfrak{g}\left(\left(u^{-1}\right)\right)$ being the field of fractions of $u^{-1} \mathfrak{g}\left[\left[u^{-1}\right]\right]$. We will ignore these mathematical subtleties and always allow for infinite power series, implicitly assuming that we are working in some suitable topological completions of the considered algebras. For a more mathematical treatment we refer the reader to e.g. [30].
} 


\section{Relations to Standard Algebras}

In this section we relate the Lie bialgebra found in the previous section to standard Lie (bi)algebras. In particular we show that the Lie algebra is locally (in the space of spectral parameters) isomorphic to the loop algebra of $\mathfrak{u}(2 \mid 2)$, but the coalgebra takes a non-standard form. Furthermore we show how the complete bialgebra can be obtained from a reduction of the loop algebra based on the maximal extension $\mathfrak{h}_{+}$of $\mathfrak{p s u}(2 \mid 2)$.

5.1. Embedding into the twisted $\mathfrak{u}(2 \mid 2)$ loop algebra. In the following we shall try to express the algebra discussed in Sect. 4.3 through elements of the loop algebra of $\mathfrak{u}(2 \mid 2)\left[i u,(i u)^{-1}\right]$ which we shall expand as Laurent polynomials

$$
\overline{\mathfrak{J}}_{n}^{A}:=(i u)^{n} \overline{\mathfrak{J}}^{A} .
$$

Standard loop algebra. First of all, it is reasonable to expect that the $\mathfrak{s u}(2) \times \mathfrak{s u}(2)$ generators are not deformed:

$$
\mathfrak{R}^{a}{ }_{b}=\overline{\mathfrak{R}}_{b}^{a}, \quad \mathfrak{L}^{\alpha}{ }_{\beta}=\overline{\mathfrak{L}}^{\alpha}{ }_{\beta} .
$$

For the $\mathfrak{u}(1) \times \mathfrak{u}(1)$ generators $\mathfrak{B}, \mathfrak{C}$ acting as $\left[\overline{\mathfrak{B}}, \overline{\mathfrak{J}}^{A}\right]=[A] \mathfrak{\mathfrak { J }}^{A}$ and $\left[\overline{\mathfrak{J}}^{A}, \overline{\mathfrak{C}}\right]=0$ we allow for a rescaling

$$
\mathfrak{C}=e \overline{\mathfrak{C}}, \quad \mathfrak{B}=f \overline{\mathfrak{B}} .
$$

Finally we make the ansatz that the fermionic generators can be mixed by a general $2 \times 2$ matrix

$$
\begin{aligned}
\mathfrak{Q}^{\alpha}{ }_{b} & =a \overline{\mathfrak{Q}}^{\alpha}{ }_{b}+b \varepsilon^{\alpha \gamma} \varepsilon_{b d} \overline{\mathfrak{S}}^{d}{ }_{\gamma}, \\
\mathfrak{S}^{a}{ }_{\beta} & =d \overline{\mathfrak{S}}^{a}{ }_{\beta}+c \varepsilon^{a c} \varepsilon_{\beta \delta} \overline{\mathfrak{Q}}^{\delta}{ }_{c} .
\end{aligned}
$$

Note that the coefficients $a, \ldots, f$ are assumed to be functions of $i u$. A generator $\overline{\mathfrak{J}}$ multiplied by a function of $i u$ is understood as a generator of the loop algebra according to the identification (5.1). Thus the new generators will generically be linear combinations of generators at different levels of the loop algebra. The latter two redefinitions are obviously consistent with the undeformed $\mathfrak{s u}(2) \times \mathfrak{s u}(2)$ transformation rules.

Consider now the brackets of supercharges. For example the bracket

$$
\left\{\left(\mathfrak{Q}_{n}\right)^{\alpha}{ }_{b},\left(\mathfrak{Q}_{m}\right)^{\gamma}{ }_{d}\right\}=2 \alpha \beta \varepsilon^{\alpha \gamma} \varepsilon_{b d} \mathfrak{C}_{m+n-1}
$$

after substitution and evaluation of $\mathfrak{u}(2 \mid 2)$ brackets reads

$$
2 a b \varepsilon^{\alpha \gamma} \varepsilon_{b d}(i u)^{m+n} \overline{\mathfrak{C}}=2 \alpha \beta \varepsilon^{\alpha \gamma} \varepsilon_{b d}(i u)^{m+n-1} e \overline{\mathfrak{C}},
$$

or $a b=\alpha \beta e / i u$ for short. The brackets of supercharges thus lead to the following four constraints

$$
a d-b c=1, \quad a d+b c=e, \quad a b=\alpha \beta e / i u, \quad c d=-\alpha^{-1} \beta e / i u,
$$

which have two solutions for $b, c, d, e$ in terms of $\alpha, \beta, i u$. Furthermore we should consider the brackets of $\mathfrak{B}$, for example

$$
\left[\mathfrak{B}, \mathfrak{Q}_{b}^{\alpha}\right]=\mathfrak{Q}_{b}^{\alpha}-2 \alpha \beta \varepsilon^{\alpha \gamma} \varepsilon_{b d}(i u)^{-1} \mathfrak{S}^{d}{ }_{\gamma} .
$$


After substitution and evaluation this leads to the relation

$$
\begin{aligned}
f a \overline{\mathfrak{Q}}_{b}^{\alpha}-f b \varepsilon^{\alpha \gamma} \varepsilon_{b d} \overline{\mathfrak{S}}^{d}{ }_{\gamma}= & a \overline{\mathfrak{Q}}_{b}^{\alpha}+b \varepsilon^{\alpha \gamma} \varepsilon_{b d} \overline{\mathfrak{S}}^{d}{ }_{\gamma}-2 \alpha \beta \varepsilon^{\alpha \gamma} \varepsilon_{b d} d(i u)^{-1} \overline{\mathfrak{S}}^{d}{ }_{\gamma} \\
& -2 \alpha \beta c(i u)^{-1} \overline{\mathfrak{Q}}_{b}^{\alpha} .
\end{aligned}
$$

We can write this, together with the bracket $[\mathfrak{B}, \mathfrak{S}]$ as

$$
\begin{array}{llrl}
f a & =a-2 \alpha \beta c / i u, & & -f b=b-2 \alpha \beta d / i u, \\
f c=-c-2 \alpha^{-1} \beta a / i u, & -f d=-d-2 \alpha^{-1} \beta b / i u .
\end{array}
$$

All these equations are equivalent to $f e=1$ upon imposing (5.7).

Altogether we find the solution

$$
f=1 / e=\sqrt{1-\frac{4 \beta^{2}}{u^{2}}}, \quad a d=\frac{1+e}{2}, \quad b=\frac{\alpha \beta e}{i u a}, \quad c=-\frac{\alpha^{-1} \beta e}{i u d}
$$

The value of $a$ (or $d$ ) is not fixed; a convenient choice is given by

$$
a=\bar{\alpha} \sqrt{\frac{1+e}{2}}, \quad d=\bar{\alpha}^{-1} \sqrt{\frac{1+e}{2}}
$$

in which case the $2 \times 2$ matrix defined by the four elements $a, b, c, d$ becomes quasiorthogonal (and for $\alpha=\bar{\alpha}=1$ strictly orthogonal).

The solution shows that the algebra of Sect. 4.3 can be embedded into the algebra of functions $\mathbb{C} /\{0\} \rightarrow \mathfrak{u}(2 \mid 2)$ with Lie brackets canonically defined as for loop algebras. Note however, that the functions $a, \ldots, f$ are not meromorphic on $\overline{\mathbb{C}}$ and not holomorphic on $\mathbb{C} /\{0\}$ as required for an embedding into the loop algebra $\mathfrak{u}(2 \mid 2)\left[i u,(i u)^{-1}\right]$. Expanding the square roots at $u=0$ or $u=\infty$ leads to Laurent series over the levels (5.1). After the expansion the singularities of the square roots cannot be seen, and thus the proposed change of basis works only locally in the complex spectral parameter plane. As we shall see shortly, the global properties are changed.

Despite these problems, the above transformation can at least be understood as a way to show that the algebra in Sect. 4.3 satisfies Jacobi identities because $\mathfrak{u}(2 \mid 2)$ does. One might work with the above $\mathfrak{u}(2 \mid 2)$-manifest basis, but in the bialgebra this would lead to a rather complicated r-matrix. In the basis of Sect. 4.3 the r-matrix takes almost the same form as for $\mathfrak{u}(2 \mid 2)\left[i u,(i u)^{-1}\right]$, but at the cost of slightly deformed Lie brackets.

Twisted loop algebra. In order to understand the global structure of the spectral parameter plane let us introduce a transformation that removes the square root singularities

$$
z^{4}=\frac{u+2 \beta}{u-2 \beta}, \quad u=2 \beta \frac{z^{4}+1}{z^{4}-1}
$$


Using the spectral parameter $z$, the generators of the deformed loop algebra in Sect. 4.3 can be expressed through meromorphic functions $\overline{\mathbb{C}} \rightarrow \mathfrak{u}(2 \mid 2)$ as follows:

$$
\begin{aligned}
\mathfrak{C}_{n} & =(2 i \beta)^{n}\left(\frac{z^{4}+1}{z^{4}-1}\right)^{n} \frac{z^{4}+1}{2 z^{2}} \overline{\mathfrak{C}}, \\
\mathfrak{B}_{n} & =(2 i \beta)^{n}\left(\frac{z^{4}+1}{z^{4}-1}\right)^{n} \frac{2 z^{2}}{z^{4}+1} \overline{\mathfrak{B}}, \\
\left(\mathfrak{Q}_{n}\right)^{\alpha}{ }_{b} & =(2 i \beta)^{n}\left(\frac{z^{4}+1}{z^{4}-1}\right)^{n} \varepsilon_{b c}\left(+\frac{1}{2} z \overline{\mathfrak{Q}}_{+}^{c \alpha}+\frac{1}{2} z^{-1} \overline{\mathfrak{Q}}_{-}^{c \alpha}\right), \\
\left(\mathfrak{S}_{n}\right)^{a}{ }_{\beta} & =(2 i \beta)^{n}\left(\frac{z^{4}+1}{z^{4}-1}\right)^{n} i \alpha^{-1} \varepsilon_{\beta \gamma}\left(-\frac{1}{2} z \overline{\mathfrak{Q}}_{+}^{a \gamma}+\frac{1}{2} z^{-1} \overline{\mathfrak{Q}}_{-}^{a \gamma}\right),
\end{aligned}
$$

as well as $\mathfrak{R}_{n}=(i u)^{n} \overline{\mathfrak{R}}, \mathfrak{L}_{n}=(i u)^{n} \overline{\mathfrak{L}}$, and where $\overline{\mathfrak{Q}}_{ \pm}^{a \beta}$ are the linear combinations

$$
\overline{\mathfrak{Q}}_{ \pm}^{a \beta}=-\bar{\alpha} \varepsilon^{a c} \overline{\mathfrak{Q}}^{\beta}{ }_{c} \mp i \alpha \bar{\alpha}^{-1} \varepsilon^{\beta \gamma} \overline{\mathfrak{S}}^{a}{ }_{\gamma} .
$$

Curiously, the embedding is into the $\mathbb{Z}_{4}$-invariant part of the $\mathbb{Z}_{4}$-automorphism of $\overline{\mathbb{C}} \rightarrow \mathfrak{u}(2 \mid 2)$ defined by the gradings

$$
[\overline{\mathfrak{R}}]=[\overline{\mathfrak{L}}] \equiv 0, \quad\left[\overline{\mathfrak{Q}}_{ \pm}\right] \equiv \pm 1, \quad[\overline{\mathfrak{B}}]=[\overline{\mathfrak{C}}] \equiv 2, \quad[z]=-1 .
$$

Furthermore the singularities of (5.14) in the $z$-plane are very restrictive: There are poles of arbitrary degree at eighth roots of unity. In addition, the generators $\overline{\mathfrak{Q}}_{ \pm}$admit single poles at $z=\infty, 0$, respectively, and $\overline{\mathfrak{C}}$ admits double poles at $z=\infty$ and $z=0$. In other words, the deformed algebra in Sect. 4.3 can be embedded into the $\mathbb{Z}_{4}$-twisted algebra $\mathfrak{u}(2 \mid 2)\left[z, z^{-1},\left(z-e^{\pi i \mathbb{Z} / 4}\right)^{-1}\right] / \mathbb{Z}_{4}$. If one furthermore allows at most double poles at $z=0, \infty$ the algebras become isomorphic.

5.2. Maximally extended algebra $\mathfrak{h}_{+}$. We now show that the complete Lie bialgebra can be obtained as a reduction of the standard loop algebra of the maximal extension $\mathfrak{h}_{+}$of $\mathfrak{p s u}(2 \mid 2)$.

Loop Bialgebra. The maximal central extension $\mathfrak{h}$ of $\mathfrak{p} \mathfrak{s u}(2 \mid 2)$ can be adjoined by its external $\mathfrak{s l}(2)$ automorphism $[49,11]$

$$
\mathfrak{h}_{+}=\mathfrak{s l}(2) \ltimes \mathfrak{p s u}(2 \mid 2) \ltimes \mathbb{R}^{3} .
$$

The automorphisms $\mathfrak{B}^{\mathfrak{a}} \mathfrak{b}$ obey the brackets

$$
\begin{aligned}
{\left[\mathfrak{B}^{\mathfrak{a}}{ }_{\mathfrak{b}}, \mathfrak{B}^{\mathfrak{c}}\right] } & =\delta_{\mathfrak{b}}^{\mathfrak{c}} \mathfrak{B}^{\mathfrak{a}} \mathfrak{d}-\delta_{\mathfrak{d}}^{\mathfrak{a}} \mathfrak{B}^{\mathfrak{c}}{ }_{\mathfrak{b}}, \\
{\left[\mathfrak{B}^{\mathfrak{a}}{ }_{\mathfrak{b}}, \mathfrak{Q}^{c \delta \mathfrak{e}}\right] } & =\delta_{\mathfrak{b}}^{\mathfrak{e}} \mathfrak{Q}^{c \delta \mathfrak{a}}-\frac{1}{2} \delta_{\mathfrak{b}}^{\mathfrak{a}} \mathfrak{Q}^{c \delta \mathfrak{e}}, \\
{\left[\mathfrak{B}^{\mathfrak{a}}\right.} & \left.\mathfrak{a}_{\mathfrak{b}}, \mathfrak{C}^{\mathfrak{c}}\right]=\delta_{\mathfrak{b}}^{\mathfrak{c}} \mathfrak{C}^{\mathfrak{a}}-\delta_{\mathfrak{d}}^{\mathfrak{a}} \mathfrak{C}^{\mathfrak{c}}{ }_{\mathfrak{b}},
\end{aligned}
$$

where we combined the supercharges $\mathfrak{Q}^{\alpha}{ }_{b}, \mathfrak{S}^{a}{ }_{\beta}$ into one doublet of generators $\mathfrak{Q}^{a \beta \mathfrak{c}}$,

$$
\mathfrak{Q}^{a \beta 1}=\varepsilon^{a c} \mathfrak{Q}_{c}^{\beta}, \quad \mathfrak{Q}^{a \beta 2}=\varepsilon^{\beta \gamma} \mathfrak{S}^{a}{ }_{\gamma},
$$


and the charges $\mathfrak{C}, \mathfrak{P}, \mathfrak{K}$ into one triplet $\mathfrak{C}_{\mathfrak{b}}^{\mathfrak{a}}$ with

$$
\mathfrak{C}^{1}{ }_{1}=-\mathfrak{C}^{2}{ }_{2}=\mathfrak{C}, \quad \mathfrak{C}^{1}{ }_{2}=\mathfrak{P}, \quad \mathfrak{C}^{2}{ }_{1}=-\mathfrak{K} .
$$

Consequently the bracket of combined supercharges reads

$$
\left\{\mathfrak{Q}^{a \beta \mathfrak{c}}, \mathfrak{Q}^{d \epsilon \mathfrak{f}}\right\}=-\varepsilon^{\beta \epsilon} \varepsilon^{\mathfrak{c f}} \varepsilon^{a k} \mathfrak{R}^{d}{ }_{k}+\varepsilon^{a d} \varepsilon^{\mathfrak{c} \mathfrak{f}} \varepsilon^{\beta \kappa} \mathfrak{L}^{\epsilon}{ }_{\kappa}+\varepsilon^{a d} \varepsilon^{\beta \epsilon} \varepsilon^{\mathfrak{c k}} \mathfrak{C}_{\mathfrak{k}}
$$

For $\mathfrak{h}_{+}$there is a non-degenerate invariant supersymmetric bilinear form, so we can write down the quadratic Casimir invariant

$$
\begin{aligned}
\mathcal{T}_{\mathfrak{h}_{+}} & =\frac{1}{2} \mathfrak{R}^{c}{ }_{d} \mathfrak{R}^{d}{ }_{c}-\frac{1}{2} \mathfrak{L}^{\gamma}{ }_{\delta} \mathfrak{L}^{\delta}{ }_{\gamma}-\frac{1}{2} \mathfrak{B}^{\mathfrak{c}}{ }_{\mathfrak{d}} \mathfrak{C}^{\mathfrak{d}}{ }_{\mathfrak{c}}-\frac{1}{2} \mathfrak{C}^{\mathfrak{d}}{ }_{\mathfrak{c}} \mathfrak{B}^{\mathfrak{c}}{ }_{\mathfrak{d}}-\frac{1}{2} \varepsilon_{a d} \varepsilon_{\beta \epsilon} \varepsilon_{\mathfrak{c} \mathfrak{f}} \mathfrak{Q}^{a \beta \mathfrak{c}} \mathfrak{Q}^{d \epsilon \mathfrak{f}} \\
& =\mathcal{T}_{\mathfrak{p} \mathfrak{s u}(2 \mid 2)}-\frac{1}{2} \mathfrak{B}^{\mathfrak{c}}{ }_{\mathfrak{d}} \mathfrak{C}^{\mathfrak{d}}{ }_{\mathfrak{c}}-\frac{1}{2} \mathfrak{C}^{\mathfrak{c}} \mathfrak{B}^{\mathfrak{d}}{ }_{\mathfrak{c}},
\end{aligned}
$$

where $\mathcal{T}_{\mathfrak{p s u}(2 \mid 2)}$ is the $\mathfrak{p s u ( 2 | 2 )}$ Casimir defined in (4.3). One might also add a term proportional to $\mathfrak{C}^{2}=\frac{1}{2} \mathfrak{C}_{\mathfrak{d}}^{\mathfrak{c}} \mathfrak{C}_{\mathfrak{c}}^{\mathfrak{d}}$ which is obviously central. The loop algebra of $\mathfrak{h}_{+}$ therefore has the following standard classical r-matrix:

$$
r_{\mathfrak{h}_{+}}=r_{\mathfrak{p s u}(2 \mid 2)}-\sum_{m=0}^{\infty}\left(\mathfrak{B}_{-1-m}\right)^{\mathfrak{c}} \mathfrak{d} \otimes\left(\mathfrak{C}_{m}\right)^{\mathfrak{d}}{ }_{\mathfrak{c}}-\sum_{m=0}^{\infty}\left(\mathfrak{C}_{-1-m}\right)^{\mathfrak{c}} \mathfrak{d} \otimes\left(\mathfrak{B}_{m}\right)^{\mathfrak{d}}{ }_{\mathfrak{c}}
$$

following the construction outlined in Sect. 2. Henceforth, the r-matrix satisfies the $\mathrm{CYBE}$ and the corresponding Lie bialgebra is quasi-triangular.

Reduction of the algebra. To make contact to physics we want to work on the fundamental representation of $\mathfrak{h}$. It is easily seen that the automorphisms cannot be realised on the fundamental representation, hence $r_{\mathfrak{h}_{+}}$cannot produce the desired fundamental r-matrix. Nevertheless there is a reduction of the algebra which leads to the desired r-matrix. Mathematically, the reduction consists in two steps: First we restrict the automorphisms to a particular subalgebra. The corresponding subalgebra of $\mathfrak{h}_{+}$has an ideal consisting of linear combinations of the charges which we then project out. The resulting algebra is the one introduced in Sect. 4.3.

In particular, we restrict the automorphisms $\left(\mathfrak{B}_{n}\right)^{\mathfrak{a}_{\mathfrak{b}}}$ to a subalgebra spanned by the linear combinations

$$
\mathfrak{B}_{n}=\left(\mathfrak{B}_{n}\right)^{1}{ }_{1}-\left(\mathfrak{B}_{n}\right)^{2}{ }_{2}+2 \alpha^{-1} \beta\left(\mathfrak{B}_{n-1}\right)^{1}{ }_{2}+2 \alpha \beta\left(\mathfrak{B}_{n-1}\right)^{2}{ }_{1} .
$$

The brackets of these automorphisms with the supercharges agree with those derived in (4.8). Furthermore, the brackets with the charges $\left(\mathfrak{C}_{n}\right)^{\mathfrak{a}}{ }_{\mathfrak{b}}$ read

$$
\begin{aligned}
{\left[\mathfrak{B}_{m},\left(\mathfrak{C}_{n}\right)^{1}{ }_{1}-\left(\mathfrak{C}_{n}\right)^{2}{ }_{2}\right] } & =-4 \alpha^{-1} \beta\left(\mathfrak{C}_{m+n-1}\right)^{1}{ }_{2}+4 \alpha \beta\left(\mathfrak{C}_{m+n-1}\right)^{2}{ }_{1}, \\
{\left[\mathfrak{B}_{m},\left(\mathfrak{C}_{n}\right)^{1}{ }_{2}\right] } & =+2\left(\mathfrak{C}_{m+n}\right)^{1}{ }_{2}-2 \alpha \beta\left(\mathfrak{C}_{m+n-1}\right)^{1}{ }_{1}+2 \alpha \beta\left(\mathfrak{C}_{m+n-1}\right)^{2}{ }_{2}, \\
{\left[\mathfrak{B}_{m},\left(\mathfrak{C}_{n}\right)^{2}{ }_{1}\right] } & =-2\left(\mathfrak{C}_{n+m}\right)^{2}{ }_{1}+2 \alpha^{-1} \beta\left(\mathfrak{C}_{m+n-1}\right)^{1}{ }_{1}-2 \alpha^{-1} \beta\left(\mathfrak{C}_{m+n-1}\right)^{2}{ }_{2} .
\end{aligned}
$$

It can be seen that the following linear combinations of the charges span an ideal of the subalgebra:

$$
\beta\left(\mathfrak{C}_{n}\right)^{1}{ }_{1}-\beta\left(\mathfrak{C}_{n}\right)^{2}{ }_{2}-\alpha^{-1}\left(\mathfrak{C}_{n+1}\right)^{1}{ }_{2}, \quad \beta\left(\mathfrak{C}_{n}\right)^{1}{ }_{1}-\beta\left(\mathfrak{C}_{n}\right)^{2}{ }_{2}-\alpha\left(\mathfrak{C}_{n+1}\right)^{2}{ }_{1}
$$


We project out this ideal analogously to the projection which turns $\mathfrak{u}(n \mid n)$ into $\mathfrak{p} \mathfrak{u}(n \mid n)$ or $\mathfrak{s u}(n \mid n)$ into $\mathfrak{p s u}(n \mid n)$. The remaining charges will be denoted by $\mathfrak{C}_{n}$ defined through

$$
\left(\mathfrak{C}_{n}\right)^{1}{ }_{1}=-\left(\mathfrak{C}_{n}\right)^{2}{ }_{2}=\mathfrak{C}_{n}, \quad\left(\mathfrak{C}_{n}\right)^{1}{ }_{2}=2 \alpha \beta \mathfrak{C}_{n-1}, \quad\left(\mathfrak{C}_{n}\right)^{2}{ }_{1}=2 \alpha^{-1} \beta \mathfrak{C}_{n-1}
$$

This step makes the brackets of supercharges coincide with (4.10) and furthermore the charges $\mathfrak{C}_{n}$ become central

$$
\left[\mathfrak{B}_{n}, \mathfrak{C}_{m}\right]=0 .
$$

In conclusion, the reduction of the algebra leads to the algebra discussed in Sect. 4.3.

Reduction of the coalgebra. One can convince oneself that the standard r-matrix $r_{\mathfrak{h}_{+}}$ for $\mathfrak{h}_{+}$contains terms which are not part of the reduced algebra. We solve this problem by modifying the r-matrix slightly before performing the reduction of the algebra. The modified r-matrix will not be meaningful in the original algebra, but it will complete the reduced algebra to a quasi-triangular bialgebra.

Before we perform the reduction of the algebra we twist the r-matrix according to (4.18) with $\mathfrak{J}:=\left(\mathfrak{C}_{-1}\right)^{1}{ }_{1}$ and $\mathfrak{J}^{\prime}:=\left(\mathfrak{B}_{0}\right)^{1}{ }_{1}-\left(\mathfrak{B}_{0}\right)^{2}{ }_{2}$,

$$
r:=r_{\mathfrak{h}_{+}}+\mathfrak{J} \wedge \mathfrak{J}^{\prime}=r_{\mathfrak{h}_{+}}+\left(\mathfrak{C}_{-1}\right)^{1}{ }_{1} \wedge\left(\left(\mathfrak{B}_{0}\right)^{1}{ }_{1}-\left(\mathfrak{B}_{0}\right)^{2}{ }_{2}\right)
$$

It is clear that $\left[\mathfrak{J}, \mathfrak{J}^{\prime}\right]=0$, and because $\mathfrak{J}^{\prime}$ is a Cartan element we also have $\left[\mathfrak{J}^{\prime}, r_{\mathfrak{h}_{+}}\right]=0$. However it turns out that

$$
\left[\mathfrak{J}, r_{\mathfrak{h}_{+}}\right]=\left(\mathfrak{C}_{-1}\right)^{1}{ }_{2} \wedge\left(\mathfrak{C}_{-1}\right)^{2}{ }_{1},
$$

and therefore the twisted r-matrix $r$ does not satisfy the CYBE according to (4.19),

$$
[[r, r]]=-\mathfrak{J}^{\prime} \wedge \wedge\left[\mathfrak{J}, r_{\mathfrak{h}_{+}}\right]=-\left(\left(\mathfrak{B}_{0}\right)^{1}{ }_{1}-\left(\mathfrak{B}_{0}\right)^{2}{ }_{2}\right) \wedge\left(\mathfrak{C}_{-1}\right)^{1}{ }_{2} \wedge\left(\mathfrak{C}_{-1}\right)^{2}{ }_{1} \neq 0 .
$$

Now let us consider the reduced algebra. The projection of the central charges (5.27) has two interesting consequences: Firstly, it makes the combination in (5.30) vanish, $\left[\mathfrak{J}, r_{\mathfrak{h}_{+}}\right]=0$ and thus it reinstates the CYBE $[[r, r]]=0$. Secondly, the twisted r-matrix can be written as

$$
r=r_{\mathfrak{p s u}(2 \mid 2)}-\sum_{m=-1}^{\infty} \mathfrak{B}_{-1-m} \otimes \mathfrak{C}_{m}-\sum_{m=+1}^{\infty} \mathfrak{C}_{-1-m} \otimes \mathfrak{B}_{m},
$$

i.e. all the undesired combinations of $\left(\mathfrak{B}_{n}\right)^{\mathfrak{a}_{\mathfrak{b}}}$ which are not part of the reduced algebra have dropped out. This classical r-matrix fully agrees with our proposal (4.13) including the shifted bounds of the sums. Thus the reduced bialgebra is identical to the one considered in Sect. 4.3.

A similar construction may be possible for the exceptional loop algebra of $\mathfrak{d}(2,1 ; \varepsilon)$ in the limit $\varepsilon \rightarrow 0$. It is worth pursuing this question further. 


\section{Different Classical Limits}

In this section we investigate the behaviour of the R-matrix in different limits. Recall that for ordinary Yangians the R-matrix depends only on one variable, the difference of the spectral parameters. Requiring that $\mathcal{R} \rightarrow 1 \otimes 1$ basically defines this limit uniquely: the difference of spectral parameters must approach infinity. To investigate the classical limit one introduces an unphysical scaling parameter $\hbar$ such that the spectral parameters scale like $u \sim \hbar^{-1}$. In contradistinction, our R-matrix does not only depend on the spectral parameters, but also on the physical coupling constant $g$. In the previous sections we used $\hbar=g^{-1}$ as a natural scale, and let the spectral parameter scale like $u \sim \hbar^{-1}$. However, there are other consistent ways of rescaling $u$ and $g$ by functions of $\hbar$, which makes it possible to have several well-defined classical limits. This is reminiscent of the AdS/CFT correspondence, which has a classical limit at strong and at weak coupling. It is even possible to define different classical limits within the strong or weak coupling regime, respectively. Our algebraic framework might make it possible to find other interesting classical limits or even to classify them.

For a different classical limit we define the natural scale by $g=\hbar^{-\kappa}$ with $\kappa<1$, and let the spectral parameters scale as usual, $u \sim \hbar^{-1}$, and also $x \sim \hbar^{-1}$. Similarly, we could let $x \sim \hbar$ which would lead to qualitatively the same results. Thus we are in the weak coupling regime for $\kappa<0$ and in the strong coupling regime for $0<\kappa<1$; nevertheless the limit will not make a distinction between positive and negative $\kappa$. We introduce the rescaled spectral parameter

$$
\tilde{u}=u \hbar .
$$

We also choose as the phase factor the same as in (3.17), leading to a classical r-matrix taking the same form as given at the top of Table 1, but with the coefficients taking the values

$$
\begin{aligned}
\frac{1}{2} A_{12}+\frac{1}{2} B_{12}=\frac{1}{2} D_{12}+\frac{1}{2} E_{12}=\frac{\tilde{\gamma}_{2}}{\tilde{\gamma}_{1}} H_{12}=\frac{\tilde{\gamma}_{1}}{\tilde{\gamma}_{2}} K_{12} & =\frac{1}{i \tilde{u}_{1}-i \tilde{u}_{2}}, \\
\frac{1}{2} A_{12}-\frac{1}{2} B_{12}=\frac{1}{2} D_{12}-\frac{1}{2} E_{12} & =-\frac{1}{4 i \tilde{u}_{1}}+\frac{1}{4 i \tilde{u}_{2}}, \\
C_{12}=F_{12} & =0, \\
G_{12}=-L_{12} & =+\frac{1}{4 i \tilde{u}_{1}}+\frac{1}{4 i \tilde{u}_{2}} .
\end{aligned}
$$

If we use the phase factor (3.18) instead of (3.17) we have to add to $r$ the diagonal terms $(1 \otimes 1) r_{0}$ with

$$
r_{0} \simeq \frac{1}{4 i \tilde{u}_{2}}-\frac{1}{4 i \tilde{u}_{1}} .
$$

The above r-matrix can be written compactly as

$$
r \simeq \frac{\mathcal{P}_{12}}{i \tilde{u}_{1}-i \tilde{u}_{2}}+\mathfrak{C}_{-1} \wedge \mathfrak{B}_{0},
$$

where $\mathcal{P}_{12}$ is the (graded) permutation. This action coincides with the action of the same r-matrix (4.13) discussed in Sect. 4 if one sets the parameters for the fundamental representation in (3.9) to

$$
a=\frac{1}{d}=\tilde{\gamma}, \quad b=c=0 .
$$


This fundamental representation is obviously consistent with the undeformed loop algebra $\mathfrak{u}(2 \mid 2)\left[i \tilde{u},(i \tilde{u})^{-1}\right]$ (i.e. the deformed algebra with $\left.\beta=0\right){ }^{12}$ Effectively this means that we reproduce the standard fundamental r-matrix for $\mathfrak{u}(2 \mid 2)\left[i \tilde{u},(i \tilde{u})^{-1}\right]$ with a Reshetikhin twist (4.18) which takes the form (6.4).

\section{Lift to Hopf Algebra}

Let us briefly discuss the lift of the Lie bialgebra to a Hopf algebra. We shall only consider the fundamental evaluation representation, without proving that the relations we give lead to a consistent Hopf algebra. In this case the generators act like $\mathfrak{J}_{n}^{A} \simeq(i u)^{n} \mathfrak{J}_{0}^{A}$, with $\mathfrak{J}_{0}^{A}$ in the fundamental representation of the Lie algebra. The action of most of the generators is known from (3.8),(3.9). In order to determine the action of $\mathfrak{B}$ and its commutation relations, we have made an ansatz similar to (4.11) and (4.8) with undetermined coefficients. It turns out that

$$
\mathfrak{B}\left|\phi^{a}\right\rangle=-\frac{1}{4 C}\left|\phi^{a}\right\rangle, \quad \mathfrak{B}\left|\psi^{\alpha}\right\rangle=+\frac{1}{4 C}\left|\psi^{\alpha}\right\rangle,
$$

is compatible with the commutators

$$
\begin{aligned}
& {\left[\mathfrak{B}_{m},\left(\mathfrak{Q}_{n}\right)^{\alpha}{ }_{b}\right] \simeq\left(\mathfrak{Q}_{m+n}\right)^{\alpha}{ }_{b}-\alpha \varepsilon^{\alpha \gamma} \varepsilon_{b d}\left(1+\mathcal{U}^{2}\right)\left(\mathfrak{S}_{m+n-1}\right)^{d}{ }_{\gamma},} \\
& {\left[\mathfrak{B}_{m},\left(\mathfrak{S}_{n}\right)^{a}{ }_{\beta}\right] \simeq-\left(\mathfrak{S}_{m+n}\right)^{a}{ }_{\beta}-\alpha^{-1} \varepsilon^{a c} \varepsilon_{\beta \delta}\left(1+\mathcal{U}^{-2}\right)\left(\mathfrak{Q}_{m+n-1}\right)^{\delta_{c}} .}
\end{aligned}
$$

Furthermore we can write the two additional central charges $\mathfrak{P}, \mathfrak{K}$ appearing in the commutators of alike supercharges using the charge $\mathfrak{C}$ at a lower level:

$$
\begin{aligned}
& \left\{\left(\mathfrak{Q}_{m}\right)^{\alpha}{ }_{b},\left(\mathfrak{Q}_{n}\right)^{\gamma}{ }_{d}\right\} \simeq \alpha \varepsilon^{\alpha \gamma} \varepsilon_{b d}\left(1+\mathcal{U}^{2}\right) \mathfrak{C}_{m+n-1}, \\
& \left\{\left(\mathfrak{S}_{m}\right)^{a}{ }_{\beta},\left(\mathfrak{S}_{n}\right)^{c}{ }_{\delta}\right\} \simeq-\alpha^{-1} \varepsilon^{a c} \varepsilon_{\beta \delta}\left(1+\mathcal{U}^{-2}\right) \mathfrak{C}_{m+n-1} .
\end{aligned}
$$

This would lead to the following relations between $\mathfrak{C}_{-1}$ and $\mathcal{U}^{2}$ :

$$
\mathfrak{C}_{-1} \simeq g \frac{1-\mathcal{U}^{2}}{1+\mathcal{U}^{2}}, \quad \mathcal{U}^{2} \simeq \frac{g-\mathfrak{C}_{-1}}{g+\mathfrak{C}_{-1}}
$$

Note that the above relations hold only on the fundamental evaluation representation. There may be further corrections which cannot be seen on this representation.

Next we would like to see if the generators $\mathfrak{B}_{n}$ are symmetries of the R-matrix. Due to the relation (2.6) for evaluation representations it suffices to consider $\mathfrak{B}_{0}$ and $\mathfrak{B}_{1}$. The cobrackets in Table 2 of these generators read ${ }^{13}$

$$
\begin{aligned}
& \delta\left(\mathfrak{B}_{0}\right)=-\alpha^{-1} \varepsilon^{b d} \varepsilon_{\alpha \gamma}\left(\mathfrak{Q}_{-1}\right)^{\alpha}{ }_{b} \wedge\left(\mathfrak{Q}_{-1}\right)^{\gamma}{ }_{d}+\alpha \varepsilon^{\beta \delta} \varepsilon_{a c}\left(\mathfrak{S}_{-1}\right)^{a}{ }_{\beta} \wedge\left(\mathfrak{S}_{-1}\right)^{c}{ }_{\delta}, \\
& \delta\left(\mathfrak{B}_{1}\right)=\left(\mathfrak{Q}_{0}\right)^{\alpha}{ }_{b} \wedge\left(\mathfrak{S}_{0}\right)^{b}{ }_{\alpha} .
\end{aligned}
$$

There is a natural lift of the cobracket for $\mathfrak{B}_{1}$ to a coproduct. One merely has to add the standard coproduct and introduce proper braiding factors for $\mathfrak{Q}$ and $\mathfrak{S}$,

$$
\begin{gathered}
\Delta\left(\mathfrak{B}_{1}\right)=\mathfrak{B}_{1} \otimes 1+1 \otimes \mathfrak{B}_{1}+\frac{1}{2} g^{-1}\left(\mathfrak{Q}_{0}\right)^{\alpha}{ }_{b} \mathcal{U}^{-1} \otimes\left(\mathfrak{S}_{0}\right)^{b}{ }_{\alpha} \\
+\frac{1}{2} g^{-1}\left(\mathfrak{S}_{0}\right)^{a}{ }_{\beta} \mathcal{U}^{+1} \otimes\left(\mathfrak{Q}_{0}\right)^{\beta}{ }_{a} .
\end{gathered}
$$

\footnotetext{
12 In other words, the $\beta=0$ undeformed algebra is a contraction of the $\beta=1$ deformed algebra, where one scales the level- $n$ generators $\mathfrak{J}_{n}$ by $\epsilon^{n}$ and takes the $\epsilon \rightarrow 0$ limit.

13 Note that $\sum_{k=1}^{-1} f_{k}=-f_{0}$.
} 
This coproduct was proposed very recently and independently in [50] where it was also shown to be a symmetry of the fundamental S-matrix. Here, we confirm this result which gives further confidence that our Lie bialgebra has a lift to a quasi-triangular double Yangian with the known fundamental R-matrix. We furthermore expect that the four new fermionic coproducts proposed in the conclusions of [50] are linear combinations of the coproducts of our generators $\mathfrak{Q}_{0,1}$ and $\mathfrak{S}_{0,1}$. The reason for the discrepancy lies in different assumed algebra relations, (7.2) in our case and those related to the representation discussed in Sect. 4.1 for [50].

Finding a coproduct for $\mathfrak{B}_{0}$ is however not as easy: According to (3.5) the grading of the two terms in $\delta\left(\mathfrak{B}_{0}\right)$ is \pm 2 whereas the grading of $\mathfrak{B}_{0}$ should be zero. This mismatch leads to inconsistencies in the braiding with $\mathcal{U}$ and to a failure of coassociativity in the coproduct. It is currently not clear how to resolve this issue. To have a coproduct for all $\mathfrak{B}_{n}$ is nevertheless important for the lift of the classical r-matrix to a universal R-matrix.

\section{Conclusions and Outlook}

In this paper we have proposed a quasi-triangular Lie bialgebra whose underlying Lie algebra is a deformation of the loop algebra of $\mathfrak{u}(2 \mid 2)$. Its classical r-matrix on the fundamental evaluation representation coincides with the classical limit of the S-matrix of [51] obtained in [26]. This bialgebra is almost the standard rational loop bialgebra based on $\mathfrak{u}(2 \mid 2)$, but there are two crucial differences: Firstly, the cobrackets include some additional non-standard terms, even for some level-0 generators. Secondly, not all Lie brackets have a uniform level: there is mixing between the levels. In comparison to the Hopf algebra symmetries of the S-matrix $[9,10,17]$ which use the three-dimensional universal central extension of $\mathfrak{p s u}(2 \mid 2)$, here we have only one central extension $\mathfrak{C}$. The three central elements of $\mathfrak{p s u}(2 \mid 2) \ltimes \mathbb{R}^{3}$ clearly have no dual partners (automorphisms) which would be needed for the standard construction of the classical r-matrix. Conversely, our central element $\mathfrak{C}$ can be paired with the $\mathfrak{u}(1)$ automorphism $\mathfrak{B}$ of $\mathfrak{u}(2 \mid 2)$ as in the proposal of [29], and we can construct a classical r-matrix. The additional central charges appear in our algebra as the central charge at a different loop level. Furthermore, the $\mathfrak{u}(1)$ automorphism does not act diagonally on the roots, and mixes the levels of the loop algebra. Due to these features the Lie algebra does not coincide with the standard $\mathfrak{u}(2 \mid 2)$ loop algebra, but to some extent the two can be related, see Sect. 5.1. Apart from that and unlike in [29], our bialgebra resembles the standard one for Yangian doubles. Since [29] reproduces the correct classical r-matrix on the fundamental representation, it would be interesting to find out if their proposal and ours are equivalent. For instance, one might compare the two when acting on bound states [52-55,11]. Whether or not they are equivalent, we believe that our approach is more suitable to find the quantisation of the bialgebra to a double Yangian Hopf algebra. The latter should be equipped with a universal R-matrix, which should be almost of the standard form for DY(u(2|2)). Again, the crucial difference will be the behaviour of the $\mathfrak{u}(1)$ automorphism and the level-mixing due to the identifications between the central charges, the braiding and the loop variable. We have made first steps in this direction in the previous section, where we have found a hidden symmetry of the fundamental R-matrix which was independently discovered in the recent paper [50] (which appeared while we were preparing our manuscript). However there are some unresolved issues concerning the full quantum braiding for the coproduct of one remaining generator. It would furthermore be interesting to see if contact with quantum deformations and the exceptional Lie superalgebra $\mathfrak{d}(2,1 ; \alpha)$ [56-61], or, on a different account, with the loop algebras encountered in the context of 
a twistor formulation of $\mathcal{N}=4$ SYM $[62,63]$, can be made. It would also be desirable to understand the bialgebra structure for other kinematical regimes such as giant magnons [64] and the near-flat limit $[65,27,28]$ and how they are related to our near plane wave setup. Concerning the algebraic determination of the dressing phase, we find no constraint for the classical r-matrix. However, quasi-triangularity for the universal R-matrix leads to stronger constraints and may allow a derivation from first principles.

Acknowledgement. We would like to thank Valentina Forini, Peter Koroteev, Tristan McLoughlin, Jan Plefka, Alessandro Torrielli and the CMP referee for interesting discussions and useful comments.

Open Access This article is distributed under the terms of the Creative Commons Attribution Noncommercial License which permits any noncommercial use, distribution, and reproduction in any medium, provided the original author(s) and source are credited.

\section{References}

1. Minahan, J.A., Zarembo, K.: The Bethe-ansatz for $\mathcal{N}=4$ super Yang-Mills. JHEP 0303, 013 (2003)

2. Beisert, N., Kristjansen, C., Staudacher, M.: The Dilatation Operator of $\mathcal{N}=4$ Conformal Super YangMills Theory. Nucl. Phys. B664, 131 (2003)

3. Bena, I., Polchinski, J., Roiban, R.: Hidden symmetries of the $A d S_{5} \times S^{5}$ superstring. Phys. Rev. D69, 046002 (2004)

4. Beisert, N., Staudacher, M.: The $\mathcal{N}=4$ SYM Integrable Super Spin Chain. Nucl. Phys. B670, 439 (2003)

5. Beisert, N., Staudacher, M.: Long-Range PSU(2,2/4) Bethe Ansaetze for Gauge Theory and Strings. Nucl. Phys. B727, 1 (2005)

6. Staudacher, M.: The factorized S-matrix of CFT/AdS. JHEP 0505, 054 (2005)

7. Beisert, N.: The su(2/2) dynamic S-matrix. http://arxiv.org/abs/hep-th/0511082, 2005

8. Arutyunov, G., Frolov, S., Plefka, J., Zamaklar, M.: The Off-shell Symmetry Algebra of the Light-cone $A d S_{5} \times S^{5}$ Superstring. J. Phys. A40, 3583 (2007)

9. Gomez, C., Hernández, R.: The magnon kinematics of the AdS/CFT correspondence. JHEP 0611, 021 (2006)

10. Plefka, J., Spill, F., Torrielli, A.: On the Hopf algebra structure of the AdS/CFT S-matrix. Phys. Rev. D74, 066008 (2006)

11. Beisert, N.: The Analytic Bethe Ansatz for a Chain with Centrally Extended su(2/2) Symmetry. J. Stat. Mech. 07, P01017 (2007)

12. Dolan, L., Nappi, C.R., Witten, E.: Yangian symmetry in D=4 superconformal Yang-Mills theory. In: Argyres, P.C. et al. (ed.), Quantum Theory and Symmetries, World Scientific, Singapore, 2004

13. Dolan, L., Nappi, C.R., Witten, E.: A Relation Between Approaches to Integrability in Superconformal Yang-Mills Theory. JHEP 0310, 017 (2003)

14. Serban, D., Staudacher, M.: Planar $\mathcal{N}=4$ gauge theory and the Inozemtsev long range spin chain. JHEP 0406, 001 (2004)

15. Agarwal, A., Rajeev, S.G.: Yangian symmetries of matrix models and spin chains: The dilatation operator of $\mathcal{N}=4$ SYM. Int. J. Mod. Phys. A20, 5453 (2005)

16. Zwiebel, B.I.: Yangian symmetry at two-loops for the $\operatorname{su}(2 / 1)$ sector of $\mathcal{N}=4$ SYM. J. Phys. A40, 1141 (2007)

17. Beisert, N.: The S-Matrix of AdS/CFT and Yangian Symmetry, PoS Solvay, 002 (2007)

18. Arutyunov, G., Frolov, S., Zamaklar, M.: The Zamolodchikov-Faddeev algebra for $A d S_{5} \times S^{5}$ superstring. JHEP 0704, 002 (2007)

19. Gomez, C., Hernández, R.: Quantum deformed magnon kinematics. JHEP 0703, 108 (2007)

20. Young, C.A.S.: q-Deformed Supersymmetry and Dynamic Magnon Representations. J. Phys. A40, 9165 (2007)

21. Beisert, N., Zwiebel, B.I.: On Symmetry Enhancement in the psu(1,1/2) Sector of $\mathcal{N}=4$ SYM. JHEP 0710, 031 (2007)

22. Janik, R.A.: The $A d S_{5} \times S^{5}$ superstring worldsheet S-matrix and crossing symmetry. Phys. Rev. D73, 086006 (2006)

23. Beisert, N., Hernández, R., López, E.: A Crossing-Symmetric Phase for $A d S_{5} \times S^{5}$ Strings. JHEP 0611, 070 (2006)

24. Beisert, N., Eden, B., Staudacher, M.: Transcendentality and crossing. J. Stat. Mech. 07, P01021 (2007)

25. Khoroshkin, S.M., Tolstoi, V.N.: Yangian double. Lett. Math. Phys. 36, 373 (1996)

26. Torrielli, A.: Classical r-matrix of the su(2/2) SYM spin-chain. Phys. Rev. D75, 105020 (2007) 
27. Klose, T., McLoughlin, T., Roiban, R., Zarembo, K.: Worldsheet scattering in $A d S_{5} \times S^{5}$. JHEP 0703, 094 (2007)

28. Klose, T., McLoughlin, T., Minahan, J.A., Zarembo, K.: World-sheet scattering in $A d S_{5} \times S^{5}$ at two loops. JHEP 0708, 051 (2007)

29. Moriyama, S., Torrielli, A.: A Yangian Double for the AdS/CFT Classical r-matrix. JHEP 0706, 083 (2007)

30. Chari, V., Pressley, A.: A guide to quantum groups. Cambridge: Cambridge University Press, 1994

31. Drinfeld, V.G.: Quantum groups. J. Math. Sci. 41, 898 (1988)

32. Beisert, N.: The Dilatation Operator of $\mathcal{N}=4$ Super Yang-Mills Theory and Integrability. Phys. Rept. 405, 1 (2004)

33. Martins, M.J., Melo, C.S.: The Bethe ansatz approach for factorizable centrally extended S-matrices. Nucl. Phys. B785, 246 (2007)

34. Arutyunov, G., Frolov, S., Staudacher, M.: Bethe ansatz for quantum strings. JHEP 0410, 016 (2004)

35. Beisert, N., Tseytlin, A.A.: On Quantum Corrections to Spinning Strings and Bethe Equations. Phys. Lett. B629, 102 (2005)

36. Hernández, R., López, E.: Quantum corrections to the string Bethe ansatz. JHEP 0607, 004 (2006)

37. Gromov, N., Vieira, P.: The $A d S_{5} \times S^{5}$ superstring quantum spectrum from the algebraic curve. Nucl. Phys. B789, 175 (2008)

38. Frolov, S., Plefka, J., Zamaklar, M.: The $A d S_{5} \times S^{5}$ superstring in light-cone gauge and its Bethe equations. J. Phys. A39, 13037 (2006)

39. Berenstein, D., Maldacena, J.M., Nastase, H.: Strings in flat space and pp waves from $\mathcal{N}=4$ Super Yang Mills. JHEP 0204, 013 (2002)

40. Parnachev, A., Ryzhov, A.V.: Strings in the near plane wave background and AdS/CFT. JHEP 0210, 066 (2002)

41. Callan, C.G. Jr., Lee, H.K., McLoughlin, T., Schwarz, J.H., Swanson, I., Wu, X.: Quantizing string theory in $A d S_{5} \times S^{5}$ : Beyond the pp-wave. Nucl. Phys. B673, 3 (2003)

42. Frolov, S., Tseytlin, A.A.: Multi-spin string solutions in $A d S_{5} \times S^{5}$. Nucl. Phys. B668, 77 (2003)

43. Beisert, N., Minahan, J.A., Staudacher, M., Zarembo, K.: Stringing Spins and Spinning Strings. JHEP 0309, 010 (2003)

44. Kruczenski, M.: Spin chains and string theory. Phys. Rev. Lett. 93, 161602 (2004)

45. Kazakov, V.A., Marshakov, A., Minahan, J.A., Zarembo, K.: Classical/quantum integrability in AdS/CFT. JHEP 0405, 024 (2004)

46. Arutyunov, G., Frolov, S.: On $A d S_{5} \times S^{5}$ string S-matrix. Phys. Lett. B639, 378 (2006)

47. Beisert, N., Kazakov, V., Sakai, K., Zarembo, K.: The Algebraic Curve of Classical Superstrings on $A d S_{5} \times S^{5}$. Commun. Math. Phys. 263, 659 (2006)

48. Reshetikhin, N.: Multiparameter quantum groups and twisted quasitriangular Hopf algebras. Lett. Math. Phys. 20, 331 (1990)

49. Serganova, V.V.: Automorphisms of simple Lie superalgebras. Math. USSR Izv. 24, 539 (1985)

50. Matsumoto, T., Moriyama, S., Torrielli, A.: A Secret Symmetry of the AdS/CFT S-matrix. JHEP 0709, 099 (2007)

51. Beisert, N.: On the scattering phase for $A d S_{5} \times S^{5}$ strings. Mod. Phys. Lett. A22, 415 (2007)

52. Dorey, N.: Magnon bound states and the AdS/CFT correspondence. J. Phys. A39, 13119 (2006)

53. Chen, H.-Y., Dorey, N., Okamura, K.: On the scattering of magnon boundstates. JHEP 0611, 035 (2006)

54. Roiban, R.: Magnon bound-state scattering in gauge and string theory. JHEP 0704, 048 (2007)

55. Chen, H.-Y., Dorey, N., Okamura, K.: The asymptotic spectrum of $\mathcal{N}=4$ super Yang-Mills spin chain. JHEP 0703, 005 (2007)

56. Zhang, R.B.: A two-parameter quantization of osp(4/2). J. Phys. A25, L991 (1992)

57. Yamane, H.: Quantized enveloping algebras associated with simple Lie superalgebras and their universal $R$-matrices. Publ. Res. Math. Inst. Sci. 1, 15 (1994)

58. Yamane, H.: On defining relations of affine Lie superalgebras and affine quantized universal enveloping superalgebras. Publ. Res. Math. Inst. Sci. 3, 321 (1999)

59. Yamane, H.: A central extension of $U_{q}\left(s l(2 \mid 2)^{(1)}\right)$ and $R$-matrices with a new parameter. J. Math. Phys. 11, $5450(2003)$

60. Heckenberger, I., Spill, F., Torrielli, A., Yamane, H.: Drinfeld second realization of quantum affine superalgebras of $D^{(1)}(2,1 ; x)$ via the Weyl groupoid. http://arxiv.org/abs/0705.1071, 2007

61. Beisert, N., Koroteev, P.: Quantum Deformations of the One-Dimensional Hubbard Model. J. Phys. A41, 255204 (2008)

62. Wolf, M.: On hidden symmetries of a super gauge theory and twistor string theory. JHEP 0502, 018 (2005)

63. Popov, A.D., Wolf, M.: Hidden symmetries and integrable hierarchy of the $\mathcal{N}=4$ supersymmetric Yang-Mills equations. Commun. Math. Phys. 275, 685 (2007) 
64. Hofman, D.M., Maldacena, J.M.: Giant magnons. J. Phys. A39, 13095 (2006)

65. Maldacena, J., Swanson, I.: Connecting giant magnons to the pp-wave: An interpolating limit of $A d S_{5} \times S^{5}$. Phys. Rev. D76, 026002 (2007)

Communicated by M.R. Douglas 